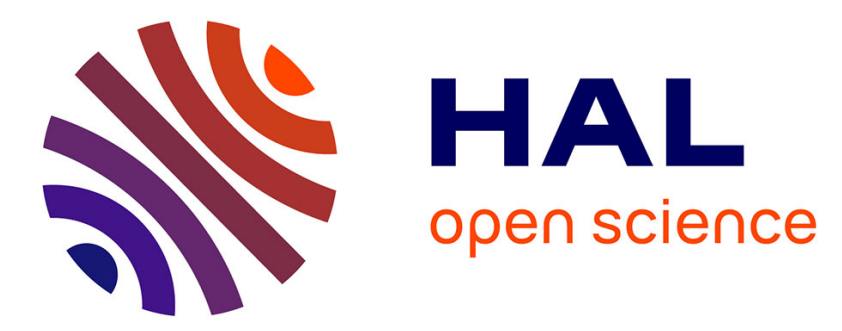

\title{
An approach for modeling three-phase piezoelectric composites
}

\author{
Raúl Guinovart-Díaz, Reinaldo Rodríguez-Ramos, Yoanh Espinosa-Almeyda, \\ Juan Carlos López-Realpozo, Serge Dumont, Frédéric Lebon, Aura Conci
}

\section{- To cite this version:}

Raúl Guinovart-Díaz, Reinaldo Rodríguez-Ramos, Yoanh Espinosa-Almeyda, Juan Carlos LópezRealpozo, Serge Dumont, et al.. An approach for modeling three-phase piezoelectric composites. Mathematical Methods in the Applied Sciences, 2017, Mathematical Modeling in Micromechanics, 40 (9), pp.3230-3248. 10.1002/mma.3937 . hal-01694029

\section{HAL Id: hal-01694029 \\ https://hal.science/hal-01694029}

Submitted on 1 Jun 2018

HAL is a multi-disciplinary open access archive for the deposit and dissemination of scientific research documents, whether they are published or not. The documents may come from teaching and research institutions in France or abroad, or from public or private research centers.
L'archive ouverte pluridisciplinaire HAL, est destinée au dépôt et à la diffusion de documents scientifiques de niveau recherche, publiés ou non, émanant des établissements d'enseignement et de recherche français ou étrangers, des laboratoires publics ou privés. 


\title{
An approach for modeling three-phase piezoelectric composites
}

\author{
Raúl Guinovart-Díaz ${ }^{\text {a*t }}$, Reinaldo Rodríguez-Ramos ${ }^{\mathrm{a}}$, \\ Yoanh Espinosa-Almeydab ${ }^{\mathrm{b}}$, Juan Carlos López-Realpozo ${ }^{\mathrm{a}}$, Serge Dumontc, \\ Frédéric Lebon' and Aura Conci ${ }^{\mathrm{d}}$
}

In this work, three-phase piezoelectric fibrous composites distributed in a parallelepiped cell is studied. The statement of the mathematical problems and the formulation of the local problems by means of the asymptotic homogenization method are presented. Closed-form formulae are obtained for the effective properties of the composites for different configurations of the cells. The present method for thick and thin mesophases can provide a point of reference for comparisons with other numerical and approximate methods.

Keywords: homogenization; determination of effective properties; elastic materials

\section{Introduction}

The detailed investigation of physical and geometric structures of the composites and the main characteristics of their components have been investigated by many theoretical and experimental works during the recent decade. An example is represented by the composite piezoelectric because of its application of sensors and actuators, its mechanic active control of the structures (elastic deformations, vibrations) or its effect induced by means of the interaction on the electric and mechanical fields.

Different micromechanical models of piezoelectric composite materials have been applied for understanding the coupling behavior between the electric and mechanic field and to predict the electroelastic effective properties (for example, Benveniste [1], Zhang et al., [2], Jiang et al., [3], Dinzart and Sabar [4], Rodríguez-Ramos et al., [5], Xu et al., [6] and Eynbeygi and Aghdam [7]). An appropriate description of the coupling phenomena produced for piezoelectric-reinforced materials is often of interest, including non-well bonded reinforcement (Wang and Pan [8], Gu et al., [9]) or coated inclusions (Shen et al., [10]; Shiah et al., [11]; Xiao et al., [12]) added in the matrix. Both cases represent interesting approximations of imperfections that occur in the contact region between the fibers and the matrix.

In this work, a third phase between the matrix and the fiber is considered for a better understanding of the idealization of the complex phenomenon that occurs at the interface. This third phase is taken as a thin layer that represents the transition zone (interphase or mesophase) between the fiber and the matrix (Jiang and Cheung, [13], Guinovart-Díaz et al., [14, 15]; Yan et al. [16], etc). The mesophase can be produced because of the chemical interaction between the constituents or it can be introduced intentionally to protect the fibers from the direct influence of the matrix.

Many works associated with coated inclusions modeling by three-phase or multiphase piezoelectric composites can be found in the literature. For example: Jiang and Cheung [13] obtained through a self-consistent approach an analytical solution of a threephase piezoelectric cylinder composite under antiplane shear and in-plane electric field applied at infinity, and they showed the non-monotonic dependence on the effective moduli of the fiber and the shear modulus. Sudak [17] analyzed elliptic piezoelectric inclusion coated using an intermediate layer in an unlimited matrix containing the electroelastic stresses and concluded that the intermediate layer produces considerable effects on the electronic performance of devices. Yang and Gao [18] used the complex variable method, in which the complex potentials are given in the power series form. They investigated the electro-elastic fields in an infinite

\footnotetext{
a Facultad de Matemática y Computación, Universidad de la Habana, San Lázaro y L, Vedado, Habana 4, CP10400, Cuba

$b^{b}$ Instituto de Ingeniería y Tecnología, Universidad Autónoma de Ciudad Juárez, Av. Del Charro 610 Norte Cd. Juárez, Chih 32310, Mexico

' Laboratoire de Mécanique et d'Acoustique, Université Aix-Marseille, CNRS, Centrale Marseille, 4 Impasse Nikola Tesla CS 4000613453 Marseille Cedex 13, France

$d^{\prime}$ Instituto de Computação, Universidade Federal Fluminense, Av. Gal. Milton Tavares de Souza s/n ${ }^{\circ}$ CEP: 24210-346, Niterói, Rio de Janeiro, Brasil

* Correspondence to: Raul Guinovart-Díaz, Facultad de Matemática y Computación, Universidad de la Habana, San Lázaro y L, Vedado, Habana 4, CP10400, Cuba.

† E-mail:guino@matcom.uh.cu
} 
matrix with N coated-piezoelectric inclusions. Yan et al., [16] applied the eigenfunction expansion-variational method to the antiplane electroelastic behavior of three-phase piezoelectric composites with doubly periodic microstructures. Moreover, Guinovart-Díaz et al., [15] show only the plane analytical expressions of the effective piezoelectric properties for three-phase composites. In this case, hexagonal and square arrays of the cells are studied. Recently, Rodríguez-Ramos et al., [19] derived the piezoelectric effective properties for three-phase composites, but for the antiplane problems, and a comparison with the spring model and finite element method (FEM) approaches is given.

The main aim of this contribution is the determination of the whole set of effective properties for three-phase piezoelectric fiber unidirectional reinforced composite with parallelepiped cell symmetry. Two approaches are used for the investigation of the macroscopic behavior of the composites. The asymptotic homogenization method (AHM) and the Finite Element Method (FEM) are applied to the aforementioned composites. The explicit derivation of the solution of the plane and antiplane local problems are given. The FEM formulation for three-phase composite is an extension of the idea developed by Nitsche [20], Becker et al. [21], and Dumont et al. [22], in which it is possible to discretize this problem using standard FEM with standard error estimate. The estimation of the effective piezoelectric moduli taking into account the fiber distribution within the matrix, the choice and permutation of the constituent phases, and the influence of the volume fractions are reported. In addition, it shows the anisotropy character of the composites induced by the distribution of the fibers arrays. The new elements of this work (as compared with previous papers, Guinovart-Díaz et al., [15, 23], Rodríguez-Ramos et al., [19]), with respect to both the AHM and FEM methods, consist in that it is the first intent to give a complete characterization by numerical (FEM) and analytical expressions (AHM) of the effective properties of the composite with a parallelepiped cell. These expressions contain the information about the constituents, fiber volume fraction, influence of the mesophase, and the periodic cell. This analysis leads to monoclinic behavior of periodic piezoelectric composites with the parallelepiped cell. The present work generalizes other relative investigations, like the aforementioned references by the previous authors of this contribution, and new ones, such as Rodríguez-Ramos et al., [5], in which effective elastic properties using three different approaches for composite materials under imperfect contact adherence are calculated. Kari et al., [24] used numerical homogenization techniques based on FEM to calculate the effective properties for different three-phase types of composites. In addition, the effects of interphase volume fraction and the material stiffness for transversely randomly distributed fiber or spherical particle composites are investigated. Sevostianov et al., [25] gave a comparative analysis of different approaches: differential approach, three-phase and spring models using AHM, to model fiber reinforced composites with imperfect interfaces and periodic square arrangement of fibers. The generalization consists in the following issues: the fiber distributions generate a parallelepiped cell in the composites and the entire sets of piezoelectric effective properties associated with plane/antiplane problems are provided, where we obtain a more general class of symmetric in the global behavior of the composite.

The outline of the paper is as follows: In Section 1, an introduction is presented. Section 2 proposes the two-scale asymptotic homogenization method and the statements of the plane and antiplane local problems. In Section 3, the finite element model is developed. Moreover, Section 4 is devoted to present some important validations of the present models. Finally, some conclusions are written.

\section{Statement of the mathematical problems and the formulation of the local problems for three-phase piezoelectric composites}

Consider a three-phase fiber piezoelectric composite as shown in Figure 1. The composite consists of a parallelepiped array of two circular and concentric infinitely long fibers of radius $R$ and $R_{1}=R+t\left(R<R_{1}\right)$, where $t$ is the thickness of mesophase. Different materials are embedded in a homogeneous medium, where the matrix (1), mesophase (I), and fiber (2) are made of piezoelectric materials or inactive materials. Also, each homogeneous phase is taken as a material with $6 \mathrm{~mm}$ symmetry. The interface conditions between the two contiguous phases occupied by $S_{s}(s=1, l, 2)$ are assumed to be in perfect contact along the interfaces $\Gamma_{1}=$ $\left\{z: \quad z=R_{1} e^{i \theta}, 0 \leq \theta \leq 2 \pi\right\}$ and $\Gamma_{2}=\left\{z: z=R e^{i \theta}, 0 \leq \theta \leq 2 \pi\right\}$. The axis of transverse symmetry coincides with the fiber direction, which is taken as the $O x_{3}$ axis. Moreover, the fibers are periodically distributed without overlapping in directions parallel to the $O w_{1}$ and $O w_{2}$-axes, where $w_{1} \neq 0$ and $w_{1} \neq 2\left(w_{2} \neq \lambda w_{1}, \lambda \in \mathbb{R}\right)$ are two complex numbers that define the parallelepiped periodic cell with angle $\theta=\measuredangle\left(w_{1}, w_{2}\right)$ of the three-phase composite (Figure 2$)$.

The coupled system of partial differential equations with coefficients rapidly oscillating for linear piezoelectric heterogeneous structures is

$$
\left(C_{i j k l}^{\varepsilon}(\mathbf{y}) u_{k, l}^{\varepsilon}+e_{k i j}^{\varepsilon}(\mathbf{y}) \phi_{, k}^{\varepsilon}\right)_{j}=0, \quad\left(e_{i k l}^{\varepsilon}(\mathbf{y}) u_{k, l}^{\varepsilon}-\kappa_{i k}^{\varepsilon}(\mathbf{y}) \phi_{, k}^{\varepsilon}\right)_{, i}=0 \quad \text { in } \quad \Omega .
$$

The equations (1) represent a system of equations for finding $u_{i}$ and $\phi$, where $u_{k}^{\varepsilon}$ and $\phi^{\varepsilon}$ are the mechanical displacement and the electric potential, respectively. The material elastic properties $C_{i j k l}$, piezoelectric $e_{k i j}$, and dielectric $\kappa_{i k}$ are piecewise functions. For simplicity, from now on, the superscript $\varepsilon$ will be omitted on the material functions that satisfy the symmetries $C_{i j k l}=C_{j i k l}=C_{k l i j}, e_{k i j}=$ $e_{k j i}, \quad \kappa_{i k}=\kappa_{k i}$. In addition, the elasticity tensor and the dielectric permittivity tensor are assumed to be positive-definite. The comma notation denotes a partial derivative relative to the $x_{j}$ component, that is, $U_{i j} \equiv \partial U / \partial x_{j}$.

For a complete solution, it is necessary to assign suitable boundary conditions, for instance

$$
u_{i}^{\varepsilon}=\hat{u}_{i} ; \quad \sigma_{i j}^{\varepsilon} n_{j}=\hat{S}_{i} ; \quad \phi^{\varepsilon}=\phi_{0} ; \quad D_{i}^{\varepsilon} n_{i}=0, \quad \text { on } \quad \partial \Omega \text {, }
$$

where $\hat{u}_{i}, \hat{S}_{i}$ and $\phi_{0}$ are the prescribed displacement, force and electric potential on the boundary of the composite. 


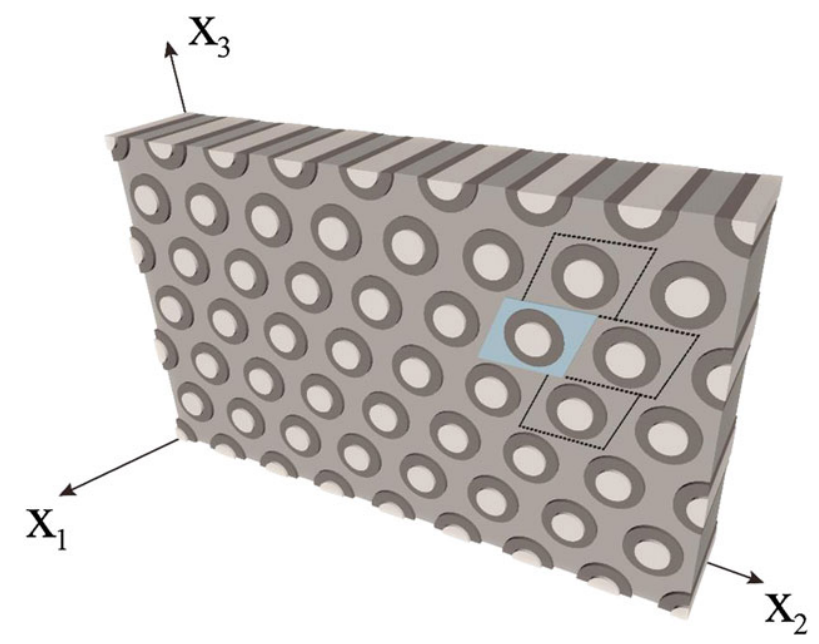

Figure 1. Three-phase periodic composite reinforced by fibers.

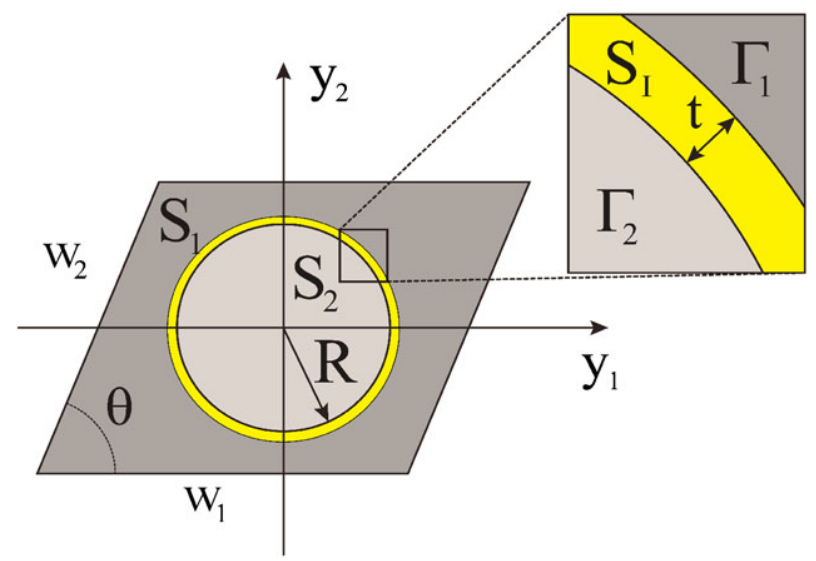

Figure 2. Composite consists of a parallelepiped array of two circular and concentric infinitely long fibers.

\subsection{Local problems}

The solution of equations (1)-(2) is represented in the form of asymptotic series in powers of the small parameter $\varepsilon$. The well-known asymptotic homogenization method reported by Rodríguez-Ramos et al., [26], as well as the local problems on the periodic cell and the effective coefficients analogous to those reported in Guinovart-Díaz et al., [14], being this, is the main aim of this work. The solutions can be solved asymptotically posing the ansatz:

$$
\begin{aligned}
& \mathbf{u}(x)=\mathbf{w}_{0}(x, y)+\varepsilon \mathbf{w}_{1}(x, y)+O\left(\varepsilon^{2}\right), \\
& \phi(x)=\phi_{0}(x, y)+\varepsilon \phi_{1}(x, y)+O\left(\varepsilon^{2}\right),
\end{aligned}
$$

and stating the two scales. The functions $\mathbf{w}_{0}, \mathbf{w}_{1}, \phi_{0}$, and $\phi_{1}$ are periodic with respect to the fast variable $y=x / \varepsilon$ and satisfy certain differential equations related to the original system. The functions $\mathbf{w}_{0}$ and $\phi_{0}$ are independent of the variable $y$, that is, $\mathbf{w}_{0}(x, y)=\mathbf{u}(x)$ and $\phi_{0}(x, y)=\phi(x)$; they are the solutions of the homogenized problem

$$
\left(C_{i j k l}^{*} u_{k, l}+e_{k i j}^{*} \phi_{, k}\right)_{, j}=0, \quad\left(e_{i k l}^{*} u_{k, l}-\kappa_{i k}^{*} \phi_{, k}\right)_{, i}=0 \quad \text { in } \Omega
$$

where $C_{i j k l}^{*}, e_{k i j}^{*}$ and $\kappa_{i k}^{*}$ denote the effective properties of the composite. Observe that original problem (1)-(2) with rapidly fluctuating coefficients are transformed in a new problem with constant coefficients and analogues boundary conditions (2) for the functions $\mathbf{u}(x)$ and $\phi(x)$.

The composite material considered in Figure 1 supposes that the heterogeneities are very small with respect to the size of the body and that they are uniformly distributed. This is a realistic assumption for a large class of applications. From a mathematical point of view, this distribution is modeled by supposing that the composite is a periodic one, and the small parameter $\varepsilon$ denote this periodicity. The multiple-scale method is well adapted to the periodic framework in which we are focused in this work. Even the first approximation of 
the solutions in problem (1)-(2) yields an error of order $\varepsilon / L$, where $L$ is a macroscopic substantial characteristic of the heterogeneous body, in comparison with the solutions of the problem (5). This value is within thousandths of the one percent for many composites with a fine scale $(\varepsilon \ll L)$ and it is sufficient in order to predict the effective properties of piezoelectric composite materials [27].

The second term in the asymptotic expansions given by (3)-(4) yields important information about the local variations of solutions, via the cell problem. The so-called local (or canonical) problems associated here with the correction terms $\mathbf{w}_{1}$ and $\phi_{1}$ to the mean variations $\mathbf{w}_{0}$ and $\phi_{0}$, respectively, because they appear in the formulae of the effective properties. Because of the linearity of the main equations, the corrections terms $\mathbf{w}_{1}$ and $\phi_{1}$ can be obtained as a linear combination of some such displacements and potentials. This, however, will not be carried out here because the main objective of this paper is the characterization of the effective properties.

Nine local problems arise, and they are denoted as ${ }_{p q} L, p L$ over the periodic unit cell, defined in the following (for instance, BravoCastillero et al., [28] and Sabina et al., [29])

The general statement of the local problems are presented in a compact form as follows:

$$
\begin{gathered}
\mathscr{F} \sigma_{i \delta, \delta}^{(s)}=0, \quad \mathscr{F} D_{\delta, \delta}^{(s)}=0 \text { in } S_{s} \\
\left\|\mathscr{F} X_{i}^{(s)}\right\|_{\gamma}=0, \quad \llbracket\left[\mathscr{F} Y^{(s)}\right]_{\gamma}=0 \text { on } \Gamma_{\gamma} \\
\left.\left.\left.\llbracket\left[\mathscr{F} \sigma_{i \delta}^{(s)} n_{\delta}\right]\right]_{\gamma}=\Psi_{\delta}^{(1)} n_{\delta}, \quad \llbracket \mathscr{F} D_{\delta}^{(s)} n_{\delta}\right]\right]_{\gamma}=\Psi_{\delta}^{(2)} n_{\delta} \text { on } \Gamma_{\gamma} \\
\left\langle\mathscr{F} X_{i}\right\rangle=0, \quad\langle\mathscr{F} Y\rangle=0
\end{gathered}
$$

Where $\mathscr{F} \sigma_{i \delta}^{(s)}=C_{i \delta k \lambda}^{(s)} X_{k, \lambda}^{(s)}+e_{\lambda i \delta}^{(s)} Y_{, \lambda}^{(s)}, \mathscr{F} D_{\delta}=e_{\delta k \lambda}^{(s)} X_{k, l}^{(s)}-\varepsilon_{0} \kappa_{\delta \lambda}^{(s)} Y_{, \lambda}^{(s)}$ and the summation convention is also understood for Greek indices, which run from 1 to 2 ; no summation is carried out over upper case indices, whether Latin or Greek. The outward unit normal vector to the interface $\Gamma_{\gamma}$ is $\mathbf{n}$. The double-bar notation $\llbracket f \rrbracket_{\gamma}$ is used to denote the jump of the relevant function $f$ across the interface $\Gamma_{\gamma}$, i.e., $\llbracket f \rrbracket_{1}=f^{(1)}-f^{(l)}$ and $\llbracket f \rrbracket_{2}=f^{(I)}-f^{(2)}$, whereas the indices (1), (I), and (2) denote the matrix, the mesophase and the fiber properties, respectively. Besides, $n_{i}$ is the component of the outward unit normal vector $\mathbf{n}$ to the interface $\Gamma_{\gamma}$. The angular brackets in (9) define the volume average per unit length over the cell, that is, $\langle F\rangle=\frac{1}{V} \int_{S} F(\mathbf{y}) d \mathbf{y}$.

From (6) to (9), it is clear that $\mathscr{F} \sigma_{i \delta}^{(s)}$ and $\mathscr{F} D_{\delta}$ are the i $\delta$-components of the stress tensor and the $\delta$-component of the electric displacement vector, associated with the displacement $\mathbf{X}^{(s)}$ and the electric potential $Y^{(s)}$.

The ${ }_{p q} L$ problem is formulated as seeking displacements ${ }_{p q} U_{i}^{(s)}(\mathbf{y})$, the electric potential ${ }_{p q} N^{(s)}(\mathbf{y})$, which are periodic of periods $w_{1}$ and $w_{2}$ and solutions of (6)-(9), where $\mathscr{F}=p q$ and the values for $\Psi_{\delta}^{(1)}, \Psi_{\delta}^{(2)}, X_{k, \lambda}^{(s)}$ and $Y_{k, \lambda}^{(s)}$ are shown in Tables I and II. The $p L$ problem is similarly stated for functions ${ }_{p} P_{i}^{(s)}(\mathbf{y})$ and ${ }_{p} Q^{(s)}(\mathbf{y})$, considering that $\mathscr{F}=p$. Only the solution of a few of these local problems is required. It depends on the-no-zero values of the properties. A more detailed explanation about this situation can be found in Rodríguez-Ramos et al. [5]).

Table I shows the specific form for the local problem boundary conditions $\Psi_{\delta}^{(1)}, \Psi_{\delta}^{(2)}$ for each local problem, and Table II illustrates solution functions $X_{k, \lambda}^{(s)}, Y_{k, \lambda}^{(s)}$, to be found for each local problem.

The homogenized (effective) moduli are given by the following expressions:

$$
\begin{aligned}
C_{i j p q}^{*} & =\left\langle C_{i j p q}+C_{i j k l p q} U_{k, l}+e_{k i j p q} N_{, k}\right\rangle, e_{i p q}^{*}=\left\langle e_{i p q}+e_{i k l p q} U_{k, l}-\varepsilon_{0} \kappa_{i k p q} N_{, k}\right\rangle, \\
e_{p i j}^{*} & =\left\langle e_{p i j}+C_{i j k l} P_{k, l}+e_{k i j p} Q_{, k}\right\rangle, \quad \varepsilon_{0} \kappa_{i p}^{*}=\left\langle\varepsilon_{0} \kappa_{i p}-e_{i k l} p P_{k, l}+\varepsilon_{0} \kappa_{i k} Q_{, k}\right\rangle,
\end{aligned}
$$

where the star denotes the overall property.

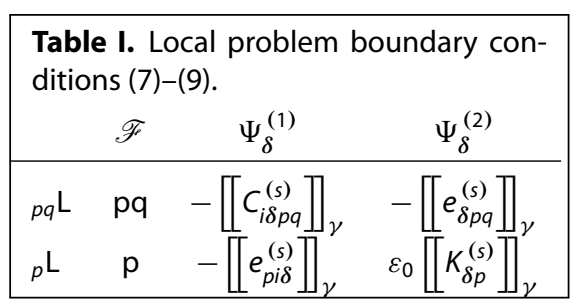

\begin{tabular}{|lcc|}
\hline \multicolumn{3}{|l|}{ Table II. Local problem } \\
boundary & conditions \\
(7)-(9). & \\
$\mathscr{F}$ & $X_{k, \lambda}^{(s)}$ & $Y_{k, \lambda}^{(s)}$ \\
\hline $\mathrm{pq}$ & $\mathrm{pq}_{k, \lambda}^{(s)}$ & ${ }_{\mathrm{pq}} N_{, \lambda}^{(s)}$ \\
$\mathrm{p}$ & ${ }_{\mathrm{p}} P_{k, \lambda}^{(s)}$ & ${ }_{\mathrm{p}} Q_{, \lambda}^{(s)}(y)$ \\
\hline
\end{tabular}




\begin{tabular}{|lllcllc|}
\hline \multicolumn{7}{|c|}{ Table III. Electroelastic material properties. } \\
Epoxy 1 & Epoxy 2 & PZT-5 & PZT (7A) & BaTiO $_{3}$ \\
\hline $\mathrm{C}_{11}(\mathrm{GPa})$ & 8 & 4.445 & 133.91 & 148 & 150.4 \\
$\mathrm{C}_{12}(\mathrm{GPa})$ & 4.4 & 2.189 & 68.98 & 76.2 & 65.63 \\
$\mathrm{C}_{13}(\mathrm{GPa})$ & 4.4 & 2.189 & 68.98 & 74.2 & 65.94 \\
$\mathrm{C}_{33}(\mathrm{GPa})$ & 8 & 4.445 & 133.91 & 131 & 145.5 \\
$\mathrm{C}_{44}(\mathrm{GPa})$ & 1.8 & 1.128 & 32.46 & $25.696(25.4)$ & 43.86 \\
$\mathrm{C}_{66}(\mathrm{GPa})$ & 1.8 & 1.128 & 32.46 & 35.9 & 42.37 \\
$\mathrm{e}_{31}\left(\mathrm{C} / \mathrm{m}^{2}\right)$ & 0 & 0 & -2.1 & -2.1 & -4.32 \\
$\mathrm{e}_{33}\left(\mathrm{C} / \mathrm{m}^{2}\right)$ & 0 & 0 & 9.5 & $12.3(9.5)$ & 17.4 \\
$\mathrm{e}_{15}\left(\mathrm{C} / \mathrm{m}^{2}\right)$ & 0 & 0 & 9.2 & $9.35(9.2)$ & 11.4 \\
$\mathrm{k}_{11}(\mathrm{nC} / \mathrm{Vm})$ & 0.0372 & 0.079 & 4.071 & $4.065(4.071)$ & 12.8 \\
$\mathrm{k}_{33}(\mathrm{nC} / \mathrm{Vm})$ & 0.0372 & 0.079 & 2.079 & $2.35(2.08)$ & 15.1 \\
\hline
\end{tabular}

Then, overall properties are the sum of the arithmetic mean plus the effect of the several properties mismatch at the interface between the two homogeneous phases. At this point, it is worthy to remember that the nontrivial solutions for the ${ }_{p q} L$ problems $(U, N)$ are determined by the no-null values of $C_{i j k l}^{(s)}$ and $e_{k i j}^{(s)}$; similarly, the values $P, Q,\left(p L\right.$ problems) are governed by the properties $e_{k i j}^{(s)}$ and $\varepsilon_{0} \kappa_{i j}^{(s)}$. Then, note that properties $e_{k i j}^{(s)}$ can be calculated through either one of two local problems. Overall piezoelectric coefficients (10) may be calculated from the solution of $p q$ and ${ }_{p} L$ problems; hence, these effective properties are the sum of arithmetic mean plus the effect of $e_{k i j}^{(s)}, \varepsilon_{0} \kappa_{i j}^{(s)}, C_{i j k l}^{(s)}$ discontinuities at the interface matrix-fiber.

From the structure of the local problems, whether ${ }_{p q} L$, and ${ }_{p} L$, it is possible to verify that each ones uncouples in two sets of equations. Uncoupled elastic plane-strain systems for ${ }_{p q} U_{\alpha}^{(s)},{ }_{p} P_{\alpha}^{(s)}$ and a coupled elastic antiplane-strain and electric system associating ${ }_{p q} U_{3}^{(s)}$ and ${ }_{p q} N^{(s)}$, for ${ }_{p q} L$, whereas ${ }_{p} P_{3}^{(s)}$ and ${ }_{p} Q^{(s)}$ are tied for ${ }_{p} L$. The non-vanishing components of the $C_{i j k l}^{(s)}, e_{k i j}^{(s)}, q_{k i j}^{(s)}$ and tensors lead to the non-homogeneous problems that have a non-zero solution. These correspond to five uncoupled elastic plane-strain problems ${ }_{11} L,{ }_{22} L, \quad{ }_{33} L,{ }_{12} L$ and ${ }_{3} L$ and four coupled elastic antiplane-strain and electric and magnetic potentials systems ${ }_{13} L,{ }_{23} L,{ }_{1} L$ and ${ }_{2} L$.

\subsection{Antiplane local problems ${ }_{\alpha 3} L,(\alpha=1,2)$}

The mathematical statement of local problems $\alpha_{3} L$, is now formulated. For simplicity, only the main results of the local problem ${ }_{\alpha 3} L$ are shown and the pre-index will be omitted in the expressions. The fundamental problem, which consists in finding the corresponding functions $U_{3}^{(s)} \equiv U^{(s)}$ and $N^{(s)}(s=1, l, 2)$ that satisfy the Laplace equations, the perfect conditions at the interfaces $\Gamma_{\gamma}$, and the null average over the periodic cell, takes the following form:

$$
\begin{gathered}
\left(C_{3 j k 3}^{(s)} U_{, k}^{(s)}+e_{k 3 j}^{(s)} N_{, k}^{(s)}\right)_{j}=0, \quad\left(e_{j 3 k}^{(s)} U_{, k}^{(s)}-\kappa_{j k}^{(s)} N_{, k}^{(s)}\right)_{j}=0, \quad \text { in } \quad \mathrm{S}_{s} \\
\llbracket U \rrbracket \gamma=0, \quad \llbracket N \rrbracket_{\gamma}=0, \quad \text { on } \quad \Gamma_{\gamma} \\
\llbracket \sigma_{i j} n_{j} \rrbracket_{\gamma}=-\llbracket C_{i j \alpha 3} \rrbracket_{\gamma} n_{j,} \quad \llbracket D_{j} n_{j} \rrbracket_{\gamma}=-\llbracket e_{j \alpha 3} \rrbracket_{\gamma} n_{j,} \quad \text { on } \quad \Gamma_{\gamma} \\
\langle U\rangle=\langle N\rangle=0,
\end{gathered}
$$

where $\sigma_{i j}^{(s)}=C_{i j 3 k}^{(s)} U_{, k}^{(s)}+e_{k i j}^{(s)} N_{, k}^{(s)}, \quad D_{j}^{(s)}=e_{j 3 k}^{(s)} U_{, k}^{(s)}-\kappa_{k j}^{(s)} N_{, k}^{(s)}$ and the angular bracket defines the volume average per unit length over the area $V$ of the cell, $U$ and $N$ being the local functions corresponding to the mechanical displacements and the electric potentials associated with the present local problems with $\alpha=1,2$.

Thus, the functions $U$ and $N$ are sought in such a way that they also are doubly periodic harmonic functions of the complex variable $z=y_{1}+i y_{2}$ in the parallelepiped periodic cell $S$, with the periods $w_{1}=1$ and $w_{2}=e^{i \theta}, 0<\theta \leq \pi / 2$ (Figure 2).

\subsection{Solution of the local problems $\alpha_{3} L,(\alpha=1,2)$}

The mathematical statement of the present problem is considered. Doubly periodic harmonic functions are to be found in terms of the following Laurent and Taylor expansions of harmonic functions $U$ and $N$ over the region $S_{1}$ matrix, $S_{1}$ mesophase, and $S_{2}$ fiber, 


$$
\begin{aligned}
& U^{(1)}(z)=\operatorname{Re}\left\{\frac{z}{R_{1}} a_{0}+\sum_{p=1}^{\infty} \circ\left(\frac{R_{1}}{z}\right)^{p} a_{p}+\sum_{k=1}^{\infty} \circ \sum_{p=1}^{\infty} \circ\left(\frac{z}{R_{1}}\right)^{p} \eta_{k p} a_{k}\right\}, \\
& N^{(1)}(z)=\operatorname{Re}\left\{\frac{z}{R_{1}} b_{0}+\sum_{p=1}^{\infty} \circ\left(\frac{R_{1}}{z}\right)^{p} b_{p}+\sum_{k=1}^{\infty} \circ \sum_{p=1}^{\infty} \circ\left(\frac{z}{R_{1}}\right)^{p} \eta_{k p} b_{k}\right\}, \quad \text { in } \mathrm{S}_{1} \\
& U^{(I)}(z)=\operatorname{Re}\left\{\sum_{p=1}^{\infty}{ }^{o} a_{-p}^{(l)}\left(\frac{R_{1}}{z}\right)^{p}+\sum_{p=1}^{\infty}{ }^{o} a_{p}^{(l)}\left(\frac{z}{R}\right)^{p}\right\}, N^{(I)}(z)=\operatorname{Re}\left\{\sum_{p=1}^{\infty} o^{o} b_{-p}^{(l)}\left(\frac{R_{1}}{z}\right)^{p}+\sum_{p=1}^{\infty}{ }^{o} b_{p}^{(l)}\left(\frac{z}{R}\right)^{p}\right\}, \text { in } \mathrm{S}_{\text {I }} \\
& U^{(2)}(z)=\operatorname{Re}\left\{\sum_{p=1}^{\infty}{ }^{o} c_{p}\left(\frac{z}{R}\right)^{p}\right\}, N^{(2)}(z)=\operatorname{Re}\left\{\sum_{p=1}^{\infty}{ }^{o} d_{p}\left(\frac{z}{R}\right)^{p}\right\}, \quad \text { in } S_{2}
\end{aligned}
$$

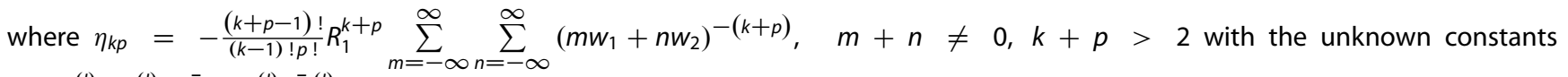
$\bar{a}_{p}, a_{p}^{(l)}, \bar{a}_{-p,}^{(l)}, \bar{b}_{p}, b_{p}^{(l)}, \bar{b}_{-p}^{(l)}, \quad c_{p}$ and $d_{p}$ are complex undetermined coefficients; $w_{1}, w_{2}$, are the periods of the parallelepiped array, respectively (Figure 2). The superscript 'o' next to the summation symbol means that ' $p$ ' runs only over odd integers so that each term in (15) has the same anti-symmetry property as $M^{(\gamma)}$ and $N^{(\gamma)}$, namely, $U^{(\gamma)}(-z)=-U^{(\gamma)}(z), N^{(\gamma)}(-z)=-N^{(\gamma)}(z)$ (see more details in the works of Bravo-Castillero et al. [28] and Sabina et al. [29]).

Substituting the previous expansion (15) into the contact conditions at the interface (12)-(13) after some algebraic manipulations, we obtain an infinite systems (Appendix A) of eight equations with the unknown constants $\bar{a}_{p}, a_{p}^{(l)}, \bar{a}_{-p}^{(l)}, \bar{b}_{p}, b_{p}^{(I)}, \bar{b}_{-p}^{(l)}, c_{p}$, and $d_{p}$. Once we have the unknown constants, we can determine the effective coefficients given in the following section.

\subsection{Antiplane effective coefficients}

The corresponding effective coefficients related to the local problems $\alpha_{3} L$, are shown.

Effective coefficients associate with the local problem ${ }_{13} L$,

$$
\begin{array}{ll}
C_{1313}^{*}=\left\langle C_{1313}\right\rangle+\left\langle C_{1331} U_{, 1}\right\rangle+\left\langle e_{113} N_{, 1}\right\rangle, & C_{2313}^{*}=\left\langle C_{2332} U_{, 2}\right\rangle+\left\langle e_{223} N_{, 2}\right\rangle, \\
e_{113}^{*}=\left\langle e_{113}\right\rangle+\left\langle e_{131} U_{, 1}\right\rangle-\left\langle\kappa_{11} N_{, 1}\right\rangle, & e_{213}^{*}=\left\langle e_{113} U_{, 2}\right\rangle-\left\langle\kappa_{11} N_{, 2}\right\rangle .
\end{array}
$$

Effective coefficients associated with the local problem ${ }_{23} L$,

$$
\begin{array}{ll}
C_{1323}^{*}=\left\langle C_{1331} U_{, 1}\right\rangle+\left\langle e_{113} N_{, 1}\right\rangle, & C_{2323}^{*}=\left\langle C_{1313}\right\rangle+\left\langle C_{1313} U_{, 2}\right\rangle+\left\langle e_{113} N_{, 2}\right\rangle, \\
e_{123}^{*}=\left\langle e_{131} U_{, 1}\right\rangle-\left\langle\kappa_{11} N_{, 1}\right\rangle, & e_{223}^{*}=\left\langle e_{223}\right\rangle+\left\langle e_{113} U_{, 2}\right\rangle-\left\langle\kappa_{11} N_{, 2}\right\rangle .
\end{array}
$$

The local functions $U$ and $N$ are the solutions of the local problems ${ }_{\alpha 3} L$, respectively. The expressions of the effective coefficients (16)(17) are transformed applying Green's theorem to the area integrals. Subsequently, using the previous expansion (15) into the lineal integrals and by the orthogonality of the system of functions $\{\cos (n x), \operatorname{sen}(n x)\}_{n=-\infty}^{\infty}$ in $[0,2 \pi]$, the analytical expressions of the dimensionless effective properties are obtained as functions of the unknown constants $\bar{a}_{1}$ and $\bar{b}_{1}$ associated with each local problem ${ }_{13} L$, or ${ }_{23} L$, as follows:

(a) in local problem ${ }_{13} L$,

$$
\begin{aligned}
C_{1313}^{*}-i C_{2313}^{*}= & \left\langle C_{1313}\right\rangle+\frac{\left(V_{1}+V_{2}\right)}{R_{1}}\left(\lambda_{1}-1\right)\left[a_{0}+\sum_{k=1}^{\infty}{ }^{o} a_{k} \eta_{k 1}+\bar{a}_{1}\right]+\frac{V_{2}}{R}\left(\lambda_{2}-\lambda_{1}\right) c_{1}+ \\
& +\frac{\left(V_{1}+V_{2}\right)}{R_{1}}\left(E_{15}^{(l)} \sqrt{\lambda_{1} \tau_{1}}-E_{15}^{(1)}\right)\left[b_{0}+\sum_{k=1}^{\infty}{ }^{o} b_{k} \eta_{k 1}+\bar{b}_{1}\right]+\frac{V_{2}}{R}\left(E_{15}^{(2)} \lambda_{2}-E_{15}^{(l)} \sqrt{\frac{\lambda_{1} \lambda_{2} \tau_{1}}{\tau_{2}}}\right) d_{1}, \\
e_{113}^{*}-i e_{213}^{*}= & \left\langle e_{113}\right\rangle+\frac{\left(V_{1}+V_{2}\right)}{R_{1}}\left(E_{15}^{(l)} \sqrt{\lambda_{1} \tau_{1}}-E_{15}^{(1)}\right)\left[a_{0}+\sum_{k=1}^{\infty}{ }^{o} a_{k} \eta_{k 1}+\bar{a}_{1}\right]+\frac{V_{2}}{R}\left(E_{15}^{(2)} \sqrt{\lambda_{2} \tau_{2}}-E_{15}^{(l)} \sqrt{\lambda_{1} \tau_{1}}\right) c_{1}- \\
& -\frac{\left(V_{1}+V_{2}\right)}{R_{1}}\left(\tau_{1}-1\right)\left[b_{0}+\sum_{k=1}^{\infty}{ }^{o} b_{k} \eta_{k 1}+\bar{b}_{1}\right]-\frac{V_{2}}{R}\left(\sqrt{\lambda_{2} \tau_{2}}-\sqrt{\frac{\lambda_{2} \tau_{1} \tau_{1}}{\tau_{2}}}\right) d_{1},
\end{aligned}
$$


(b) in local problem ${ }_{23} L$,

$$
\begin{aligned}
C_{1323}^{*}-i C_{2323}^{*}= & -i\left\langle C_{1313}\right\rangle+\left(\lambda_{1}-1\right) \frac{\left(V_{1}+V_{2}\right)}{R_{1}}\left[a_{0}+\sum_{k=1}^{\infty}{ }^{o} a_{k} \eta_{k 1}+\bar{a}_{1}\right]+\left(\lambda_{2}-\lambda_{1}\right) \frac{V_{2}}{R} c_{1}+ \\
& +\left(E_{15}^{(I)} \sqrt{\lambda_{1} \tau_{1}}-E_{15}^{(1)}\right) \frac{\left(V_{1}+V_{2}\right)}{R_{1}}\left[b_{0}+\sum_{k=1}^{\infty}{ }^{o} b_{k} \eta_{k 1}+\bar{b}_{1}\right]+\left(E_{15}^{(2)} \lambda_{2}-E_{15}^{(l)} \sqrt{\frac{\lambda_{1} \lambda_{2} \tau_{1}}{\kappa_{2}}}\right) \frac{V_{2}}{R} d_{1}, \\
e_{123}^{*}-i e_{223}^{*}= & -i\left\langle e_{113}\right\rangle+\left(E_{15}^{(l)} \sqrt{\lambda_{1} \tau_{1}}-E_{15}^{(1)}\right) \frac{\left(V_{1}+V_{2}\right)}{R_{1}}\left[a_{0}+\sum_{k=1}^{\infty}{ }^{o} a_{k} \eta_{k 1}+\bar{a}_{1}\right]+\left(E_{15}^{(2)} \sqrt{\lambda_{2} \tau_{2}}-E_{15}^{(l)} \sqrt{\lambda_{1} \tau_{1}}\right) \frac{V_{2}}{R} c_{1}- \\
& -\left(\tau_{1}-1\right) \frac{\left(V_{1}+V_{2}\right)}{R_{1}}\left[b_{0}+\sum_{k=1}^{\infty}{ }^{o} b_{k} \eta_{k 1}+\bar{b}_{1}\right]-\left(\sqrt{\lambda_{2} \tau_{2}}-\sqrt{\frac{\lambda_{2} \tau_{1} \tau_{1}}{\tau_{2}}}\right) \frac{V_{2}}{R} d_{1},
\end{aligned}
$$

(c) in local problem ${ }_{1} L$,

$$
\begin{aligned}
e_{113}^{*}-i e_{123}^{*}= & \left\langle e_{113}\right\rangle+\frac{\left(V_{1}+V_{2}\right)}{R_{1}}\left(\lambda_{1}-1\right) \sqrt{C_{1313}^{(1)} \kappa_{11}^{(1)}}\left[a_{0}+\sum_{k=1}^{\infty}{ }^{o} a_{k} \eta_{k 1}+\bar{a}_{1}\right]+\frac{V_{2}}{R} \sqrt{C_{1313}^{(1)} \kappa_{11}^{(1)}}\left(\sqrt{\lambda_{2} \tau_{2}}-\sqrt{\frac{\lambda_{1} \lambda_{1} \tau_{2}}{\lambda_{2}}}\right) c_{1}+ \\
& +\frac{\left(V_{1}+V_{2}\right)}{R_{1}}\left(E_{15}^{(1)} \sqrt{\lambda_{1} \tau_{1}}-E_{15}^{(1)}\right) \sqrt{C_{1313}^{(1)} \kappa_{11}^{(1)}}\left[b_{0}+\sum_{k=1}^{\infty}{ }^{o} b_{k} \eta_{k 1}+\bar{b}_{1}\right]+\frac{V_{2}}{R} \sqrt{C_{1313}^{(1)} \kappa_{11}^{(1)}}\left(E_{15}^{(2)} \sqrt{\lambda_{2} \tau_{2}}-E_{15}^{(1)} \sqrt{\lambda_{1} \tau_{1}}\right) d_{1}, \\
\kappa_{11}^{*}-i \kappa_{21}^{*}= & \left\langle\kappa_{11}\right\rangle-\frac{\left(V_{1}+V_{2}\right)}{R_{1}} \kappa_{11}^{(1)}\left(E_{15}^{(l)} \sqrt{\lambda_{1} \tau_{1}}-E_{15}^{(1)}\right)\left[a_{0}+\sum_{k=1}^{\infty}{ }^{o} a_{k} \eta_{k 1}+\bar{a}_{1}\right]-\kappa_{11}^{(1)} \frac{V_{2}}{R}\left(E_{15}^{(2)} \tau_{2}-E_{15}^{(1)} \sqrt{\frac{\lambda_{1} \tau_{1} \tau_{2}}{\lambda_{2}}}\right) c_{1}+ \\
& +\frac{\left(V_{1}+V_{2}\right)}{R_{1}} \kappa_{11}^{(1)}\left(\tau_{1}-1\right)\left[b_{0}+\sum_{k=1}^{\infty}{ }^{o} b_{k} \eta_{k 1}+\bar{b}_{1}\right]+\frac{V_{2}}{R} \kappa_{11}^{(1)}\left(\tau_{2}-\tau_{1}\right) d_{1},
\end{aligned}
$$

(d) in local problem ${ }_{2} L$,

$$
\begin{aligned}
e_{213}^{*}-i e_{223}^{*}= & -i\left\langle e_{223}\right\rangle+\frac{\left(V_{1}+V_{2}\right)}{R_{1}}\left(\lambda_{1}-1\right) \sqrt{C_{1313}^{(1)} \kappa_{11}^{(1)}}\left[a_{0}+\sum_{k=1}^{\infty}{ }^{o} a_{k} \eta_{k 1}+\bar{a}_{1}\right]+\frac{V_{2}}{R} \sqrt{C_{1313}^{(1)} \kappa_{11}^{(1)}}\left(\sqrt{\lambda_{2} \tau_{2}}-\sqrt{\frac{\lambda_{1} \lambda_{1} \tau_{2}}{\lambda_{2}}}\right) c_{1}+ \\
& +\frac{\left(V_{1}+V_{2}\right)}{R_{1}}\left(E_{15}^{(2)} \sqrt{\lambda_{1} \tau_{1}}-E_{15}^{(1)}\right) \sqrt{C_{1313}^{(1)} \kappa_{11}^{(1)}}\left[b_{0}+\sum_{k=1}^{\infty}{ }^{o} b_{k} \eta_{k 1}+\bar{b}_{1}\right]+\frac{V_{2}}{R} \sqrt{C_{1313}^{(1)} \kappa_{11}^{(1)}}\left(E_{15}^{(2)} \sqrt{\lambda_{2} \tau_{2}}-E_{15}^{(l)} \sqrt{\lambda_{1} \tau_{1}}\right) d_{1} \\
\kappa_{12}^{*}-i \kappa_{22}^{*}= & -i\left\langle\kappa_{22}\right\rangle-\frac{\left(V_{1}+V_{2}\right)}{R_{1}} \kappa_{11}^{(1)}\left(E_{15}^{(l)} \sqrt{\lambda_{1} \tau_{1}}-E_{15}^{(1)}\right)\left[a_{0}+\sum_{k=1}^{\infty}{ }^{o} a_{k} \eta_{k 1}+\bar{a}_{1}\right]-\frac{V_{2}}{R} \kappa_{11}^{(1)}\left(E_{15}^{(2)} \tau_{2}-E_{15}^{(l)} \sqrt{\frac{\lambda_{1} \tau_{1} \tau_{2}}{\lambda_{2}}}\right) c_{1}+ \\
& +\frac{\left(V_{1}+V_{2}\right)}{R_{1}}\left(\tau_{1}-1\right) \kappa_{11}^{(1)}\left[b_{0}+\sum_{k=1}^{\infty}{ }^{o} b_{k} \eta_{k 1}+\bar{b}_{1}\right]+\frac{V_{2}}{R} \kappa_{11}^{(1)}\left(\tau_{2}-\tau_{1}\right) d_{1} .
\end{aligned}
$$

Also, $\langle f\rangle=f^{(1)} \mathrm{V}_{1}+f^{(1)} \mathrm{V}_{1}+f^{(2)} \mathrm{V}_{2}$, where $\mathrm{V}_{1}, \mathrm{~V}_{1}$ and $\mathrm{V}_{2}$ are the volume fraction per unit length occupied by the matrix, mesophase, and the fiber, respectively. Moreover, $V_{1}+V_{1}+V_{2}=1$ and $V=\left|w_{1}\right|\left|w_{2}\right| \sin \theta$ represent the volume of periodic cell. Besides, $\lambda_{1}=C_{1313}^{(1)} / C_{1313}^{(1)}, \lambda_{2}=C_{1313}^{(2)} / C_{1313}^{(1)}, \tau_{1}=\kappa_{11}^{(1)} / \kappa_{11}^{(1)}, \tau_{2}=\kappa_{11}^{(2)} / \kappa_{11}^{(1)}, E_{15}^{(s)}=e_{113}^{(s)} / \sqrt{C_{1313}^{(s)} \kappa_{11}^{(s)}}, \quad s=1,1$, 2. The expressions (18)(21) of the effective coefficients depend on the material properties, volume fractions, and the unknown constants $a_{0}, b_{0}, a_{k}, b_{k}, c_{1}$, and $d_{1}$. The unknown constants can be found analytically from the system obtained in Appendix A.

\subsection{Analytical solution of the plane local problems ${ }_{q q} L$}

The solutions of the uncoupled elastic plane-strain systems are presented in this work because this kind of problem, to be more specific the plane problems ${ }_{q q} L$ is the one that explains the piezoelectric effect that results from a two-phase composite with one intermediate phase having elastic or piezoelectric properties. 
The mathematical statement of the plane problem in terms of the local function is the following:

$$
\begin{aligned}
\sigma_{\alpha \delta, \delta}^{(s)} & =0 \text { in } Y_{s \prime} \\
\llbracket\left[U_{i}^{(s)}\right]_{1} & =0 \text { on } \Gamma_{1} ; \llbracket\left[U_{i}^{(s)} \rrbracket\right]_{2}=0 \text { on } \Gamma_{2}, \\
\llbracket\left[\sigma_{i \delta}^{(s)} n_{\delta}\right]_{1} & =-\llbracket C_{i \delta q q} \rrbracket_{1} n_{\delta} \text { on } \Gamma_{1} ; \llbracket\left[\sigma_{i \delta}^{(s)} n_{\delta}\right]_{2}=-\llbracket C_{i \delta q q} \rrbracket_{2} n_{\delta} \text { on } \Gamma_{2}, \\
\left\langle U_{i}\right\rangle & =0
\end{aligned}
$$

where $\sigma_{11}^{(s)}=\left(k_{s}+m_{s}\right) U_{1,1}^{(s)}+\left(k_{s}-m_{s}\right) U_{2,2}^{(s)}, \sigma_{22}^{(s)}=\left(k_{s}-m_{s}\right) U_{1,1}^{(s)}+\left(k_{s}+m_{s}\right) U_{2,2}^{(s)}, \sigma_{12}^{(s)}=m_{s}\left(U_{1,2}^{(s)}+U_{2,1}^{(s)}\right)$, being

$$
k_{s}=\left(C_{1111}^{(s)}+C_{1122}^{(s)}\right) / 2, \quad I_{s}=C_{1133}^{(s)}=C_{2233}^{(s)}, \quad m_{s}=C_{1212}^{(s)}=\left(C_{1111}^{(s)}-C_{1122}^{(s)}\right) / 2
$$

The method of complex variables in terms of two harmonic functions $\varphi_{s}(z)$ and $\psi_{s}(z)$ and the Kolosov-Muskhelishvili complex potentials are applicable. The potentials are related to the displacement and stress components by means of the classical formulae:

$$
\begin{aligned}
2 m_{s}\left(U_{1}^{(s)}+i U_{2}^{(s)}\right) & =\kappa_{s} \varphi_{s}(z)-z \bar{\varphi}_{s}^{\prime}(z)-\bar{\psi}_{s}(z), \\
\sigma_{11}^{(s)}+\sigma_{22}^{(s)} & =2\left(\varphi_{s}^{\prime}(z)+\bar{\varphi}_{s}^{\prime}(z)\right), \\
\sigma_{22}^{(s)}-\sigma_{11}^{(s)}+2 i \sigma_{12}^{(s)} & =2\left(\bar{z} \varphi_{s}^{\prime \prime}(z)+s_{s}^{\prime}(z)\right),
\end{aligned}
$$

where the prime denotes a derivative with respect to $z$, the overbar is a complex conjugate, and $\kappa_{s}=3-4 v_{s}^{T}$; here, $v_{s}^{T}$ is the transverse Poisson's ratio and $s=1, l, 2$.

From equations $(22)_{2}$ and $(23)_{1}$, we obtain

$$
\begin{gathered}
\chi_{1}\left[\kappa_{1} \varphi_{1}(z)-z \bar{\varphi}_{1}^{\prime}(z)-\bar{\psi}_{1}(z)\right]=\left[\kappa_{l} \varphi_{l}(z)-z \bar{\varphi}_{l}^{\prime}(z)-\bar{\psi}_{l}(z)\right] \text { on } \Gamma_{1} \\
\chi_{2}\left[\kappa_{l} \varphi_{l}(z)-z \bar{\varphi}_{l}^{\prime}(z)-\bar{\psi}_{l}(z)\right]=\chi_{1}\left[\kappa_{2} \varphi_{2}(z)-z \bar{\varphi}_{2}^{\prime}(z)-\bar{\psi}_{2}(z)\right] \text { on } \Gamma_{2}
\end{gathered}
$$

and from $(22)_{3}$ and $(23)_{2-3}$, we can write

$$
\begin{aligned}
& z \bar{\varphi}_{1}^{\prime}(z)+\varphi_{1}(z)+\bar{\psi}_{1}(z)+\bar{z} \gamma_{1}(q)+z \gamma_{2}(q)=z \bar{\varphi}_{l}^{\prime}(z)+\bar{\psi}_{l}(z)+\varphi_{l}(z) \text { on } \Gamma_{1}, \\
& z \bar{\varphi}_{l}^{\prime}(z)+\varphi_{l}(z)+\bar{\psi}_{l}(z)+\bar{z} \gamma_{3}(q)+z \gamma_{4}(q)=z \bar{\varphi}_{2}^{\prime}(z)+\bar{\psi}_{2}(z)+\varphi_{2}(z) \text { on } \Gamma_{2},
\end{aligned}
$$

with $\chi_{1}=m_{1} / m_{1} ; \quad \chi_{2}=m_{2} / m_{1}$.

The complex potentials functions $\varphi_{s}(z)$ and $\psi_{s}(z)$ are searched for the periodic cell that contains the origin of coordinates in the following form [29]

$$
\begin{gathered}
\varphi_{1}(z)=a_{o}\left(z / R_{1}\right)+\sum_{p=1}^{\infty}{ }^{o} a_{p}\left(R_{1} / z\right)^{p}+\sum_{k=1}^{\infty} o \sum_{p=1}^{\infty}{ }^{o} a_{k} \eta_{k p}\left(z / R_{1}\right)^{p}, \\
1(z)=b_{o}\left(z / R_{1}\right)+\sum_{p=1}^{\infty}{ }^{o} b_{p}\left(R_{1} / z\right)^{p}+\sum_{k=1}^{\infty} o \sum_{p=1}^{\infty}{ }^{o} b_{k} \eta_{k p}\left(z / R_{1}\right)^{p}+\sum_{k=1}^{\infty} o \sum_{p=1}^{\infty}{ }^{o} k a_{k} R_{1}^{p+k} C_{p+k}^{p} T_{p+k}\left(z / R_{1}\right)^{p}, \\
\varphi_{l}(z)=\sum_{k=1}^{\infty}{ }^{o} c_{k}(z / R)^{k}+\sum_{k=1}^{\infty}{ }^{o} c_{-k}\left(R_{1} / z\right)^{k}, \quad \psi_{l}(z)=\sum_{k=1}^{\infty}{ }^{o} d_{k}(z / R)^{k}+\sum_{k=1}^{\infty}{ }^{o} d_{-k}\left(R_{1} / z\right)^{k}, \\
\varphi_{2}(z)=\sum_{k=1}^{\infty}{ }^{o} e_{k}(z / R)^{k}, \quad \psi_{2}(z)=\sum_{k=1}^{\infty}{ }^{o} f_{k}(z / R)^{k} .
\end{gathered}
$$

where $T_{p+k}=\sum_{m, n}^{\infty} \frac{\bar{w}_{1} m+\bar{w}_{2} n}{\left(w_{1} m+w_{2} n\right)^{p+k}} ; p+k \geq 3$ and $C_{p+k}^{p}=\frac{(p+k) !}{p ! k !}$. The double-periodicity and quasi-periodicity of these local functions $U_{1}(z)$ and $U_{2}(z)$ lead to $a_{0}=-A_{1} a_{1} R_{1}^{2}+\left[\left(A_{3}+\kappa_{1} \bar{A}_{3}\right) b_{1} R_{1}^{2} /\left(\kappa_{1}+1\right)\left(\kappa_{1}-1\right)\right]$ and $\bar{b}_{0}=\left[A_{3} \kappa_{1} a_{1}+A_{5} \bar{a}_{1}-\bar{A}_{1} \bar{b}_{1}\right] R_{1}^{2}$. Also, $A_{1}=\left(\bar{w}_{1} \delta_{2}-\bar{w}_{2} \delta_{1}\right) /\left(\bar{w}_{1} w_{2}-w_{1} \bar{w}_{2}\right), \quad A_{3}=\left(w_{1} \delta_{2}-w_{2} \delta_{1}\right) /\left(w_{1} \bar{w}_{2}-\bar{w}_{1} w_{2}\right)$ and $A_{5}=\left(w_{1} \bar{\gamma}_{2}-w_{2} \bar{\gamma}_{1}\right) /\left(\bar{w}_{1} w_{2}-w_{1} \bar{w}_{2}\right), w_{h} w_{i}$ $\delta_{i}=2 \zeta\left(w_{i} / 2\right), \gamma_{i}=2 Q\left(w_{i} / 2\right)-\bar{w}_{i} \wp\left(w_{i} / 2\right), \wp(z)$ is the double-periodic elliptic Weierstrass's function, $Q(z)$ is the meromorphic Natanzon's function related to $\wp(z)$ and the quasi-periodic Weierstrass $\zeta(z)$ function such that $\zeta^{\prime}(z)=-\wp(z)$. 
Replacing the expansions (28)-(31) into the contact conditions (24)-(27), after some convenient algebraic manipulations, we obtain the infinite systems of equations (Appendix B) with the unknown constants $a_{p}$. The complex unknown constants $a_{p}$ and their conjugate denoted by the overbar are the solutions of the corresponding local problems $q_{q} L$. The sum by the repeated indices $j, k$ and $p$ are applied with $j, k, p=1,3,5, \ldots$ and $\delta_{1 p}$ is the Kronecker's delta and the magnitudes that appear in the system are written in Appendix B. The solutions of the systems of each local problem depend on the material constants, geometry of the fibers, and the different fields related to the problems ${ }_{q q} L$. Once we have the unknown constants, we can determine the effective coefficients given in the following section.

\subsection{Effective coefficients in plane}

The corresponding effective coefficients related to the local problems $\mathscr{F} L(\mathscr{F}=q q, 12)$ are shown

(a) elastic coefficients:

$$
\begin{aligned}
& C_{11 \mathscr{F}}^{*}=\left\langle C_{11 \mathscr{F}}\right\rangle-\frac{1}{\chi_{1}} \frac{\left(V_{l}+V_{2}\right)}{m_{1} R_{1}} \operatorname{Re}\left[\llbracket m \rrbracket_{1}\left[\kappa_{l} C_{-1}-3 c_{3} \frac{R_{1}^{3}}{R^{3}}-d_{1} \frac{R_{1}}{R}\right]+\llbracket k \rrbracket_{1}\left[\left(\kappa_{l}-1\right) c_{1} \frac{R_{1}}{R}-d_{-1}\right]\right]- \\
& -\frac{\chi_{2}}{\chi_{1}} \frac{V_{2}}{m_{2} R} \operatorname{Re}\left[\llbracket m \rrbracket_{2}\left[\kappa_{l} c_{-1} \frac{R_{1}}{R}-3 c_{3}-d_{1}\right]+\llbracket k \rrbracket_{2}\left[\left(\kappa_{l}-1\right) c_{1}-d_{-1} \frac{R_{1}}{R}\right]\right] \text {, } \\
& \mathrm{C}_{22 \mathscr{F}}^{*}=\left\langle C_{22 \mathscr{F}}\right\rangle+\frac{1}{\chi_{1}} \frac{\left(V_{l}+V_{2}\right)}{m_{1} R_{1}} \operatorname{Re}\left[\llbracket m \rrbracket_{1}\left[\kappa_{l} C_{-1}-3 c_{3} \frac{R_{1}^{3}}{R^{3}}-d_{1} \frac{R_{1}}{R}\right]-\llbracket k \rrbracket_{1}\left[\left(\kappa_{l}-1\right) c_{1} \frac{R_{1}}{R}-d_{-1}\right]\right]+ \\
& +\frac{\chi_{2}}{\chi_{1}} \frac{V_{2}}{m_{2} R} \operatorname{Re}\left[\llbracket m \rrbracket_{2}\left[\kappa_{l} c_{-1} \frac{R_{1}}{R}-3 c_{3}-d_{1}\right]-\llbracket k \rrbracket_{2}\left[\left(\kappa_{l}-1\right) c_{1}-d_{-1} \frac{R_{1}}{R}\right]\right] \text {, } \\
& C_{12 \mathscr{F}}^{*}=\left\langle C_{12 \mathscr{F}}\right\rangle-\frac{\llbracket m \rrbracket_{1}}{\chi_{1}} \frac{\left(V_{l}+V_{2}\right)}{m_{1} R_{1}} \operatorname{Im}\left[\kappa_{l} C_{-1}+3 c_{3} \frac{R_{1}^{3}}{R^{3}}+d_{1} \frac{R_{1}}{R}\right]-\frac{\chi_{2} \llbracket m_{2} \rrbracket_{2}}{\chi_{1}} \frac{V_{2}}{m_{2} R} I m\left[\kappa_{l} C_{-1} \frac{R_{1}}{R}+3 c_{3}+d_{1}\right] \text {, } \\
& C_{33 \mathscr{F}}^{*}=\left\langle C_{33 \mathscr{F}}\right\rangle-\frac{\llbracket I \rrbracket_{1}}{\chi_{1}} \frac{\left(V_{l}+V_{2}\right)}{m_{1} R_{1}} \operatorname{Re}\left[\left(\kappa_{l}-1\right) c_{1} \frac{R_{1}}{R}-d_{-1}\right]-\frac{\chi_{2} \llbracket / \rrbracket_{2}}{\chi_{1}} \frac{V_{2}}{m_{2} R} \operatorname{Re}\left[\left(\kappa_{l}-1\right) c_{1}-d_{-1} \frac{R_{1}}{R}\right] \text {, }
\end{aligned}
$$

(b) piezoelectric coefficients:

$$
e_{3 \mathscr{F}}^{*}=\left\langle e_{3 \mathscr{F}}\right\rangle-\frac{\llbracket e_{311} \rrbracket_{1}}{\chi_{1}} \frac{\left(V_{1}+V_{2}\right)}{m_{1} R_{1}} \operatorname{Re}\left[\left(\kappa_{l}-1\right) c_{1} \frac{R_{1}}{R}-d_{-1}\right]-\frac{\chi_{2} \llbracket e_{311} \rrbracket_{2}}{\chi_{1}} \frac{V_{2}}{m_{2} R} \operatorname{Re}\left[\left(\kappa_{l}-1\right) c_{1}-d_{-1} \frac{R_{1}}{R}\right],
$$

where $|V|$ is the area of the periodic cell and uses the short notation. The expressions of the effective coefficients (22) and (33) are acquired after applying the Green's theorem to the area integrals in the corresponding (10) in the plane local problems $\mathscr{F} L$. Subsequently, using the previous expansion (15) into the lineal integrals and by the orthogonality of the system of functions $\{\cos (n x), \operatorname{sen}(n x)\}_{n=-\infty}^{\infty}$ in $[0,2 \pi]$, the analytical expressions of the effective properties are obtained as functions of the unknown constants $c_{1}, c_{-1}, c_{3}, d_{1}$ and $d_{-1}$ associated with each local problem $\mathscr{F} L$ and are defined in Appendix $C$.

The problem ${ }_{3} L$ contributes only with piezoelectric effective coefficients that satisfy the symmetry condition $e_{3 \mathscr{F}}^{*}=e_{\mathscr{F} 3}^{*}$ and the non-zero dielectric permittivity component given by

$$
\kappa_{33}^{*}=\left\langle\kappa_{33}\right\rangle+\frac{\llbracket e_{311} \rrbracket_{1}}{\chi_{1}} \frac{\left(V_{l}+V_{2}\right)}{m_{1} R_{1}} \operatorname{Re}\left[\left(\kappa_{l}-1\right) c_{1} \frac{R_{1}}{R}-d_{-1}\right]+\frac{\chi_{2} \llbracket e_{311} \rrbracket_{2}}{\chi_{1}} \frac{V_{2}}{m_{2} R_{1}} \operatorname{Re}\left[\left(\kappa_{l}-1\right) c_{1}-d_{-1} \frac{R_{1}}{R}\right] .
$$

\section{Finite element method}

In this section, a finite element approach is proposed in order to solve the three-phase problems. First, the uncoupled elastic planestrain problems $p q L$ can be written

$$
\begin{aligned}
\sigma_{\alpha \delta, \delta}^{(s)} & =0 \text { in } Y_{s} \\
\llbracket\left[u^{(s)}\right]_{1} & \left.=0 \text { on } \Gamma_{1}, \llbracket\left[u^{(s)}\right]\right]_{2}=0 \text { on } \Gamma_{2}, \\
{\left.\left[\sigma^{(s)}(u) n\right]\right]_{1} } & \left.=S_{1} \text { on } \Gamma_{1}, \llbracket\left[\sigma^{(s)}(u) n\right]\right]_{2}=S_{2} \text { on } \Gamma_{2}, \\
\langle u\rangle & =0
\end{aligned}
$$

where $\sigma(u)=C \varepsilon(u), S_{1}\left(\right.$ resp. $\left.S_{2}\right)$ is the jump across $\Gamma_{1}$ (resp. $\Gamma_{2}$ ) given in equation (13). 
In order to take into account the jumps in the constraint, a discontinuous Galerkin method has been developed and a weak formulation of (35) can be written as

$$
\sum_{s} \int_{Y_{s}} \sigma^{s}: \varepsilon^{s} d y+\sum_{i=1}^{2} \int_{\Gamma_{i}}\left(\left\langle\sigma^{s}(u) n\right\rangle_{i} \llbracket v \rrbracket_{i}+\llbracket u \rrbracket_{i}\left\langle\sigma^{s}(v) n\right\rangle_{i}\right) d l+\frac{\beta}{h} \int_{\Gamma_{i}} \llbracket u \rrbracket_{i} \llbracket v \rrbracket_{i} d l=-\sum_{i=1}^{2} \int_{\Gamma_{i}} S_{i}\langle v\rangle_{i} d l,
$$

where $h$ is the size of the mesh and $\beta$ is a constant (independent of the mesh) large enough to ensure the stability of the problem $[20,21])$.

Using this formulation, it is possible to discretize this problem using standard FEM with standard error estimate [20-22]).

In case of coupled antiplane problem $\alpha_{3} L_{1}(\alpha=1,2)$, it is easier to work with vanishing jumps. For that purpose, one can make the change of variables

$$
{ }_{\alpha 3} \tilde{U}^{(Y)}(\xi)={ }_{\alpha 3} U^{(Y)}(\xi)+\xi \alpha,
$$

and then, equation (13) becomes

$$
\llbracket \tilde{\sigma}_{i j} n_{j} \rrbracket_{\gamma}=0, \llbracket \tilde{D}_{j} n_{j} \rrbracket \gamma=0 \text { on } \Gamma_{\gamma}
$$

and equation (14) is transformed into

$$
\left\langle\alpha_{3} \tilde{U}^{(Y)}(\xi)\right\rangle=\left\langle\xi_{\alpha}\right\rangle ;\left\langle\alpha_{3} N^{(Y)}(\xi)\right\rangle=0
$$

Equations (11) and (12) remain unchanged.

Finally, the effective coefficients associated with the local problem ${ }_{13} L$ are defined by

$$
C_{1313}^{*}=\left\langle C_{1313} \tilde{U}_{, 1}\right\rangle+\left\langle e_{113} N_{, 1}\right\rangle, \mathrm{e}_{113}^{*}=\left\langle e_{113} \tilde{U}_{, 1}\right\rangle-\left\langle\kappa_{11} N_{, 1}\right\rangle
$$

The coefficients $C_{2313}^{*}$ and $e_{213}^{*}$ are computed as in equation (16).

Effective coefficients associated with the local problem ${ }_{23} L$ are defined by

$$
C_{2323}^{*}=\left\langle C_{1313} \tilde{U}_{, 2}\right\rangle+\left\langle e_{113} N_{, 2}\right\rangle, \mathrm{e}_{223}^{*}=\left\langle e_{113} \tilde{U}_{, 2}\right\rangle-\left\langle\kappa_{11} N_{, 2}\right\rangle
$$

and the coefficients $C_{1323}^{*}$ and $e_{123}^{*}$ are computed as in equation (17).

Let us notice that, for every small thickness of the mesophase, it is very expansive to mesh the mesophase finely because it is necessary to have neighboring elements with similar size, and consequently, the mesh has to hold a huge total number of elements (over than $10^{9}$ for $e=10^{-3}$ ). Then the results obtained with this method in the last section are realized with a rough discretization of the mesophase, leading to a lesser accuracy of the results provided by this method.

\section{Analysis of results}

The effective properties of the polymeric matrix containing piezoelectric material in homogeneities are evaluated with the proposed micromechanical models and with the finite element analysis. The electro-elastic properties of the constituent materials are shown in Table III. The polarization is assumed to be $x_{3}$ direction. The aim of this section is to point out the effects of the fiber-matrix mesophase on the piezoelectric properties of composite materials based on the asymptotic homogenization. To date, to the authors' knowledge, the problems associated with the calculations of effective properties with piezoelectric materials and inhomogeneous interphase conditions have not been proposed sufficiently in the literature. The obtained model is applied to the situation presented in Figure 3 for a composite with imperfect contact between the matrix and fiber and modeled by a thin interphase (Figure 3(a)). A composite with ring-shaped fiber considered in Xu et al. [6] (Figure 3(b)) and a three-phase composite with a thick mesophase (Figure 3(c)) are studied in detail. Some numerical results derived from the present model are given in order to illustrate the effect of the mesophase and their validation with other approaches.

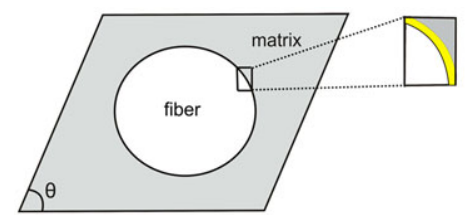

a) Thin mesophase

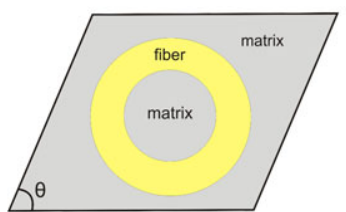

b) Ring-shaped fiber

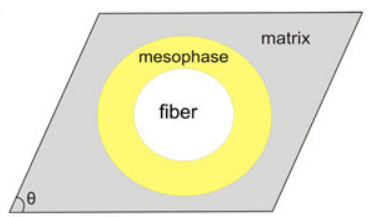

c) Three-phase composite

Figure 3. Fig. 3(a) Thin mesophase; Fig. 3(b) Ring-shaped fiber; Fig. 3(c) Thick mesophase. 
Table IV. Effective properties calculated by three methods: three phase approach (3-phases), AHM spring model and FEM, for PZT-fiber/BaTiO ${ }_{3}$-mesophase/Epoxy 1-matrix and different mesophase thickness $\eta=t / \mathrm{R}_{2}$.

\begin{tabular}{|lllcllclllll}
$\eta=t / R_{2}$ & & $C_{13}^{*}$ & $C_{33}^{*}$ & $C_{44}^{*}$ & $C_{66}^{*}$ & $\left(C_{11}^{*}+C_{12}^{*}\right) / 2$ & $e_{31}^{*}$ & $e_{33}^{*}$ & \multicolumn{1}{c}{$e_{15}^{*}$} & $\kappa_{33}^{*}$ & $\kappa_{11}^{*}$ \\
\hline \multirow{4}{*}{$10^{-1}$} & 3-phases & 8.7079 & 50.029 & 3.6054 & 4.7981 & 12.83 & -0.15722 & 7.0553 & 0.018735 & 2.2548 & 0.108 \\
& spring & 7.5038 & 40.843 & 3.1132 & 3.9228 & 10.911 & -0.093894 & 5.4118 & 0.013488 & 0.97711 & 0.086557 \\
& FEM & 8.707 & 50.029 & 4.729 & 3.605 & 12.830 & -0.157 & 7.055 & 0.0138 & 2.200 & 0.107294 \\
$10^{-2}$ & 3-phases & 7.6352 & 41.734 & 3.1631 & 4.011 & 11.116 & -0.099654 & 5.5693 & 0.013921 & 1.0994 & 0.088299 \\
& spring & 7.534 & 40.863 & 3.1221 & 3.9384 & 10.955 & -0.094341 & 5.4115 & 0.013556 & 0.97711 & 0.086556 \\
& FEM & 7.635 & 41.733 & 3.951 & 3.163 & 11.116 & -0.0997 & 5.567 & 0.014727 & 1.06800 & 0.087670 \\
$10^{-3}$ & 3-phases & 7.547 & 40.952 & 3.127 & 3.9471 & 10.975 & -0.094909 & 5.4272 & 0.013598 & 0.98928 & 0.086727 \\
& spring & 7.537 & 40.865 & 3.123 & 3.94 & 10.96 & -0.094386 & 5.4114 & 0.013563 & 0.97711 & 0.086556 \\
& FEM & 7.550 & 40.977 & 3.8890 & 3.128 & 10.980 & -0.095 & 5.425 & 0.014414 & 0.96000 & 0.086132 \\
$10^{-4}$ & 3-phases & 7.5383 & 40.874 & 3.1235 & 3.9408 & 10.962 & -0.094443 & 5.413 & 0.013567 & 0.97832 & 0.086573 \\
& spring & 7.5374 & 40.865 & 3.1231 & 3.9401 & 10.96 & -0.094391 & 5.4114 & 0.013563 & 0.97711 & 0.086556 \\
& FEM & 7.539 & 40.893 & 3.8414 & 3.124 & 10.962 & -0.094 & 5.411 & 0.014382 & 0.94900 & 0.0859492 \\
\hline
\end{tabular}
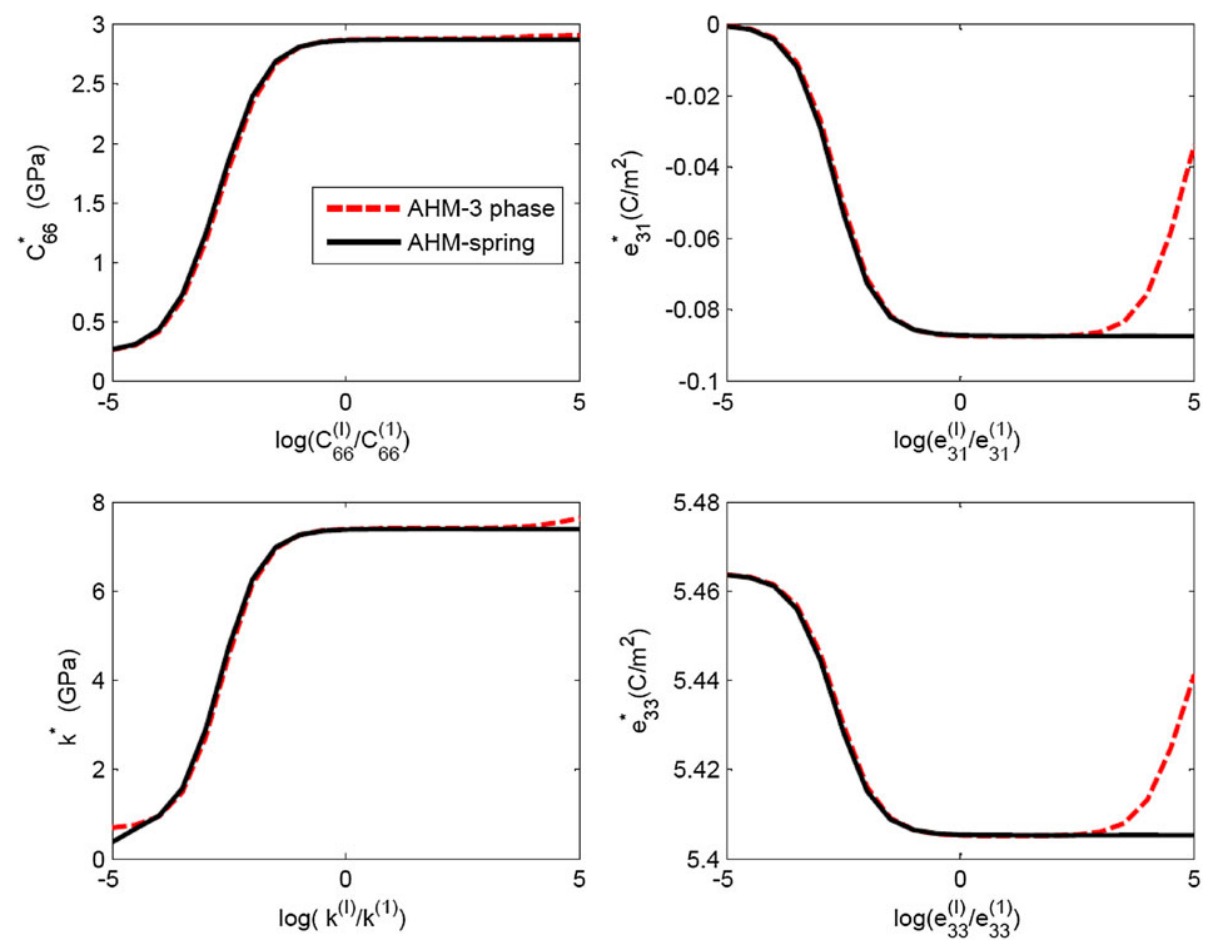

Figure 4. Comparisons between the in-plane effective properties of composite with mesophase computed using the exact three-phase solution and the mechanical imperfect model (AHM spring) in function of the properties of mesophase. AHM, asymptotic homogenization method.

(1) In order to validate the present model, some electro-elastic effective properties of a three-phase composite PZT-fiber/BaTiO ${ }_{3}$ mesophase/Epoxy 1-matrix with square periodic cells are presented in Table IV for different thickness $t$ of the mesophase. The effective properties are calculated applying two different approaches by AHM, in particular, the current model denoted by AHM 3-phase and spring model (AHM spring) reported in Rodriguez-Ramos et al., [5] and an FEM for three-phase composite (FEM) given in the expressions (40)-(41). The fiber volume fraction used in the computation is $V_{2}=0.4$. In the spring model, the imperfect parameters are considered as a function of mesophase properties given in the following form:

$$
K_{n}=\frac{E^{\prime}\left(1-v^{\prime}\right)}{\left(1+v^{\prime}\right)\left(1-2 v^{\prime}\right) t} \text { and } K_{t}=K_{s}=\frac{E^{\prime}}{2\left(1+v^{\prime}\right) t}
$$

where $E^{\prime}, v^{\prime}$ are the Young and Poisson moduli of the mesophase, respectively. The numerical results of the present three-phase model (AHM 3-phases) are close to FEM and much more proximate to the values for the imperfect model (AHM spring) when the mesophase thickness $\eta=t / R_{2}$ decreases. 

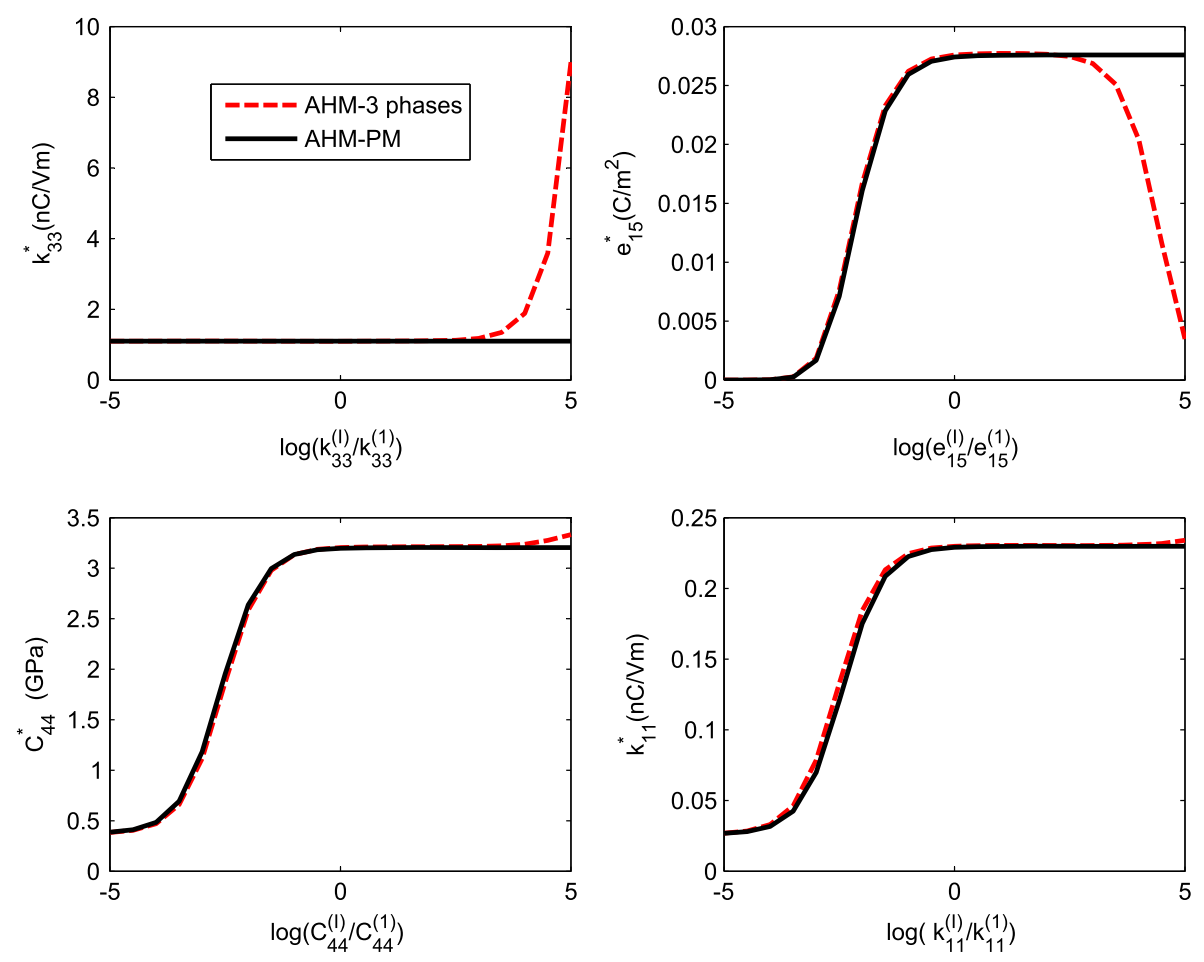

Figure 5. Comparisons between out-of-plane effective properties of composite with mesophase computed using the exact three-phase solution and the mechanical imperfect model (AHM spring) in function of the mesophase properties. AHM, asymptotic homogenization method

(2) The following figures (Figures 4 and 5) show in-plane and out-of-plane effective properties $C_{44}^{*}, C_{66}^{*}, k^{*}=\left(C_{11}^{*}+\right.$ $\left.C_{12}^{*}\right) / 2, \quad e_{31}^{*}, e_{33}^{*}, e_{15}^{*}, \kappa_{11}^{*}$ and $\kappa_{33}^{*}$ related to a unidirectional periodic isotropic transversal composite with hexagonal cell made of Epoxy 2 matrix with piezoelectric PZT-5 reinforcements, which physical characteristics are given in Table III. In the computation, the Poisson ratio of the mesophase is fixed and equal to $v^{(I)}=0.34$, the imperfect parameters considered in the AHM-spring model are given by (42), the reinforcement and mesophase volume fraction are $V_{2}=0.5$ and $V_{1}=0.001$, respectively. The existence of a thin mesophase (Figure 3a) between the matrix and fiber with thickness $t$ is considered. Figure 5 shows a comparison between the effective properties of composite with mesophase computed using the exact three-phase solution and the spring model [5] under only mechanical imperfect contact as a function of the respective properties of the mesophase value $\xi=\log \left(C_{44}^{(I)} / C_{44}^{(1)}\right)$. It is seen that for a ratio $\eta=t / R=0.001$, the two solutions are numerically indistinguishable for the coefficients except for extremely elevated values of $C_{44}^{(I)} / C_{44}^{(1)}$, where $C_{44}^{(1)} / C_{44}^{(1)}$ denotes the ratio between the mesophase property and the matrix. Furthermore, it should be noted that for a large enough value of $C_{44}^{(1)} / C_{44}^{(1)}$, the exact three-phase solution attains asymptotically to the rigid fiber limit, while the imperfect interface solution attains asymptotically to the properties of a composite with perfect contact calculated by finite element in [30]. The two figures also reveal the influence of the mesophase on the effective moduli and the range where the model of the three-phase composite is equivalent to the model of a two-phase composite under imperfect contact as reported in Rodríguez-Ramos et al. [5]. The discrepancy between three-phase and spring models via AHM is because the spring model leads to the properties of two-phase composite without mesophase. The spring model for piezoelectric composites with imperfect contact is an idealization; in fact, it requires the presence of certain additional parameters that describe the electrical imperfection between the phases. However, the three-phase model reflects the presence of electrical and mechanical barriers in the composite.

(3) In Xu et al., [6] is an analytical method using the generalized eigenstrain concept integrated with the theory of doubly quasi-periodic Riemann boundary value problem is presented. The piezoelectric composites containing the doubly periodic piezoelectric fibers with a ring-shaped cross-section under antiplane shear coupled with in-plane electric load are investigated (Figure 3(b)). In this work, motivated by the study of ring-shaped cross-section piezoelectric composites, a comparison between the out-of-plane effective electro-elastic coefficients by a three-phase model and those reported in Xu et al., [6] is given in Table V. The angle of periodic cell is $\theta=45^{\circ}$. The PZT-7A fiber occupied the mesophase region $S_{l}$ with volume fraction $V_{l}=0.1$, and the Epoxy 1 matrix is in the regions $S_{1}$ and $S_{2}$. The volume fraction in $S_{2}$ region is $V_{2}=0.4$. The material parameters used in the computation are $C_{44}^{(I)}=25.4 G \mathrm{~Pa}, \quad e_{15}^{(l)}=9.2 \mathrm{C} / \mathrm{m}^{2}$, and $\kappa_{11}^{(I)}=4.071 \mathrm{nC} / \mathrm{Vm}$. The results have very good agreements.

In Table Vl, we consider the same piezoelectric composite reported in Table V, that is, composite with ring-shaped cross-section and circular cross-section piezoelectric fibers. Here, the angle of periodic cell is $\theta=45^{\circ}$, and therefore, new effective coefficients are not null. The overall properties of the composite exhibit monoclinic symmetry. The effective properties are calculated by the ana- 


\begin{tabular}{|lccc|}
\hline \multicolumn{4}{|l|}{ Table V. Comparison between the out-of-plane effective } \\
electro-elastic coefficients for a piezoelectric fiber composite \\
(Epoxy & $1 / P Z T-7 A / E p o x y$ & 1 ), with ring-shaped cross-section \\
\multicolumn{2}{|l}{ and the periodic rhombic cell $\theta=45^{\circ}$ reported by Xu et } \\
al., [6] and the present three-phase model by AHM. \\
(GPa) & $C_{44}^{*}$ & $C_{45}^{*}=C_{54}^{*}$ & $C_{55}^{*}$ \\
\hline AHM & 3.297088984 & -0.090269997 & 3.11654899 \\
{$[6]$} & 3.297088971 & -0.090269974 & 3.11654902 \\
$\left(\mathrm{C} / \mathrm{m}^{2}\right)$ & $e_{15}^{*}$ & $e_{14}^{*}=e_{25}^{*}$ & $e_{24}^{*}$ \\
$\mathrm{AHM}$ & 0.067679261 & -0.020945162 & 0.109569585 \\
{$[6]$} & 0.067679261 & -0.020945161 & 0.109569583 \\
$(\mathrm{nC} / \mathrm{Vm})$ & $\kappa_{11}^{*}$ & $\kappa_{12}^{*}=\kappa_{21}^{*}$ & $\kappa_{22}^{*}$ \\
$\mathrm{AHM}$ & 0.090446282 & -0.009938185 & 0.110322653 \\
{$[6]$} & 0.090446283 & -0.009938185 & 0.110322652 \\
\hline
\end{tabular}

AHM, asymptotic homogenization method.

\begin{tabular}{|c|c|c|c|c|c|c|c|}
\hline$(\mathrm{GPa})$ & $C_{11}^{*}$ & $C_{12}^{*}$ & $C_{13}^{*}$ & $C_{16}^{*}$ & $C_{22}^{*}$ & $C_{23}^{*}$ & $C_{26}^{*}$ \\
\hline AHM & 11.038 & 6.218 & 6.0004 & -0.25965 & 11.119 & 6.027 & 0.21933 \\
\hline FEM & 10.913 & 6.332 & 6.0153 & -0.25804 & 11.025 & 6.034 & 0.21997 \\
\hline (GPa) & $C_{33}^{*}$ & $C_{36}^{*}$ & $C_{44}^{*}$ & $C_{45}^{*}$ & $C_{55}^{*}$ & $C_{66}^{*}$ & \\
\hline AHM & 16.763 & -0.013286 & 3.2971 & -0.09027 & 3.1165 & 2.4303 & \\
\hline FEM & 16.6515 & -0.018603 & 3.2963 & -0.09022 & 3.1158 & 2.3187 & \\
\hline$\left(\mathrm{C} / \mathrm{m}^{2}\right)$ & $e_{31}^{*}$ & $e_{32}^{*}$ & $e_{33}^{*}$ & $e_{36}^{*}$ & $e_{14}^{*}$ & $e_{15}^{*}$ & $e_{24}^{*}$ \\
\hline AHM & -0.048149 & -0.04895 & 1.0564 & 0.00040 & -0.020945 & 0.067679 & 0.10957 \\
\hline FEM & -0.048047 & -0.049167 & 1.0564 & 0.00056 & -0.020934 & 0.067688 & 0.10951 \\
\hline$(\mathrm{nC} / \mathrm{Vm})$ & $\kappa_{11}^{*}$ & $\kappa_{12}^{*}$ & $\kappa_{22}^{*}$ & $\kappa_{33}^{*}$ & & & \\
\hline AHM & 0.090446 & -0.0099382 & 0.11032 & 0.24463 & & & \\
\hline FEM & 0.088040 & -0.0093880 & 0.10683 & 0.23825 & & & \\
\hline
\end{tabular}

AHM, asymptotic homogenization method.

lytical expressions (18)-(21) and (22)-(34) denoted by AHM and FEM, respectively, reported in Section 3. The results have shown good concordance.

In Figure 6, we consider two types of piezoelectric composites, that is, composites with ring-shaped cross-section (Figure 3(b)) and circular cross-section piezoelectric fibers as in Xu et al., [6]. The fiber is PZT-7A, and the matrix is Epoxy 1. The angle of periodic cell $\theta=60^{\circ}$ (hexagonal fiber arrangement) is taken; the overall properties of the composites are transversely isotropic at this angle. Only some effective piezoelectric coefficients, that is, $e_{31}^{*}, c_{66}^{*}, e_{15}^{*}$ and $\kappa_{33}^{*}$ are shown in Figure 5 in order to illustrate the results. The model given in Xu et al., [6] and the present model AHM have good agreements for the coefficient $e_{15}^{*}$. The obtained values from the ringshaped cross-section fiber composites are very much higher than those obtained from the circular cross-section fiber under the same fiber volume fraction. The coefficients $e_{31}^{*}, c_{66}^{*}$ and $\kappa_{33}^{*}$ only are calculated by AHM. These coefficients monotonically increase in absolute values when the fiber volume fraction increases for the circular cross-section fiber composites.

In Figures 7-8, some effective properties for three-phase electro-elastic composites are presented versus the mesophase thickness $t / R$. Three different cases are considered in both figures. In Figure 7, the parallelepiped cell is fixed with the angle of periodic cell $\theta=45^{\circ}$ and the properties of the matrix and fiber are changing, the mesophase is a PZT-7A in all cases, and the circular fiber and matrix region are two Epoxies. The objective is to study the effect of the soft and hard reinforcements on the effective properties of composite. The ratio $\chi=c_{66}^{(2)} / c_{66}^{(1)}$ between the shear coefficients of both Epoxies indicates soft $(\chi=0.01)$ reinforcement, ring-shaped cross-section $(\chi=1)$, and rigid fiber $(\chi=100)$. The effect of soft or rigid circular fiber can be observed in the properties $c_{45}^{*}, e_{36}^{*}$, and $e_{15}^{*}$ in comparison with the ring-shaped fiber composite. The curves of the composite with a rigid fiber exhibit an extreme value inside of interval $[0,1]$ and notice that the extreme increases. On the other hand, in Figure 8 , it is possible to study the practical significance of having parallelepiped arrangements of the fiber distribution. Three different parallelepiped arrangements are considered, that is, $\theta=90^{\circ}, 75^{\circ}, 45^{\circ}$ for the study of the influence of parallelepiped arrays on the effective properties behavior in the composite with mesophase of PZT-7A and the circular fiber and matrix region made of Epoxy 1 . The volume fraction of the circular fiber used for the calculation is $V_{2}=0.5$. Notice the remarkable effect of the cell arrays on different overall properties as the thickness of the interface is 

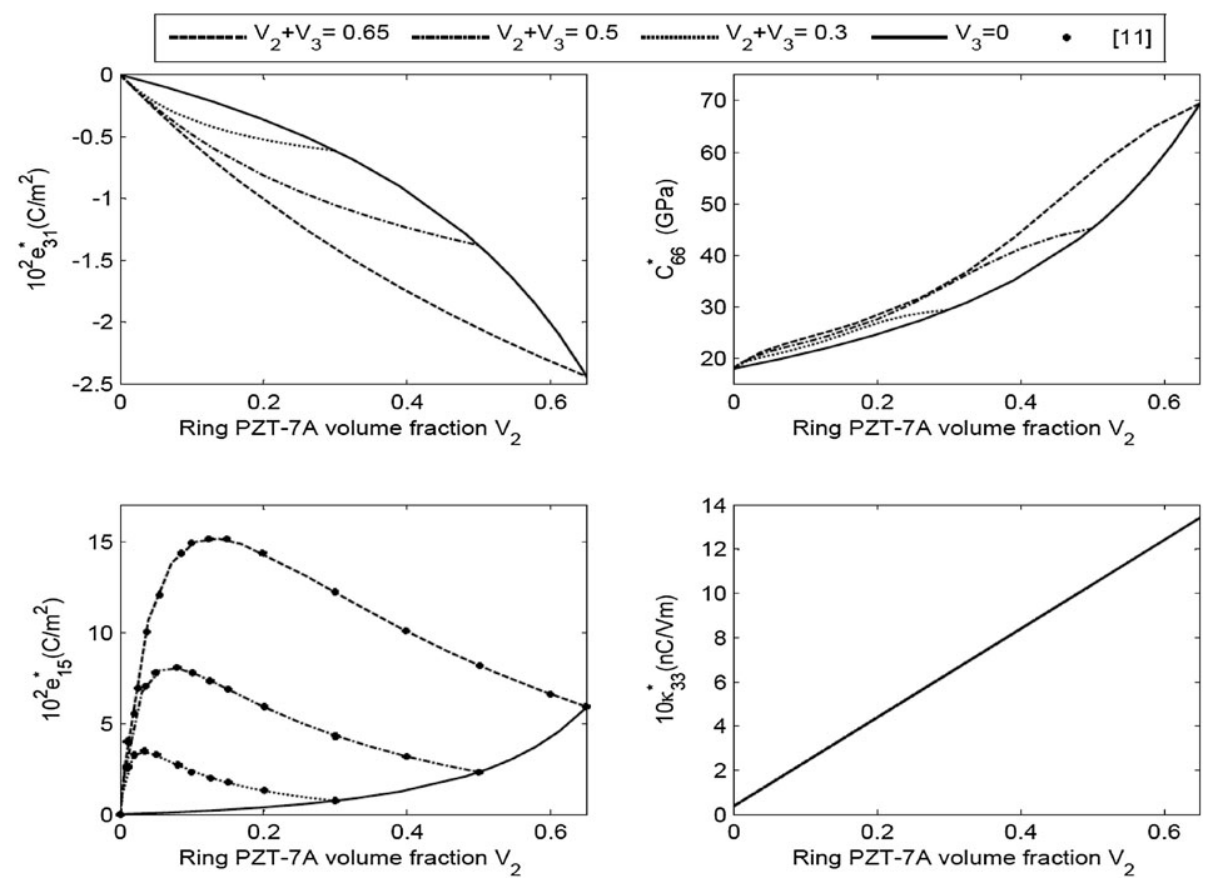

Figure 6. The effective piezoelectric coefficient $e_{31}^{*}, C_{66}^{*}, e_{15}^{*}$ and $\kappa_{33}^{*}$ for a composite with ring-shaped cross-section and hexagonal periodic cell are calculated by the present model. In particular, $e_{15}^{*}$ coincides with the equivalent coefficient reported in Xu et al., [6].
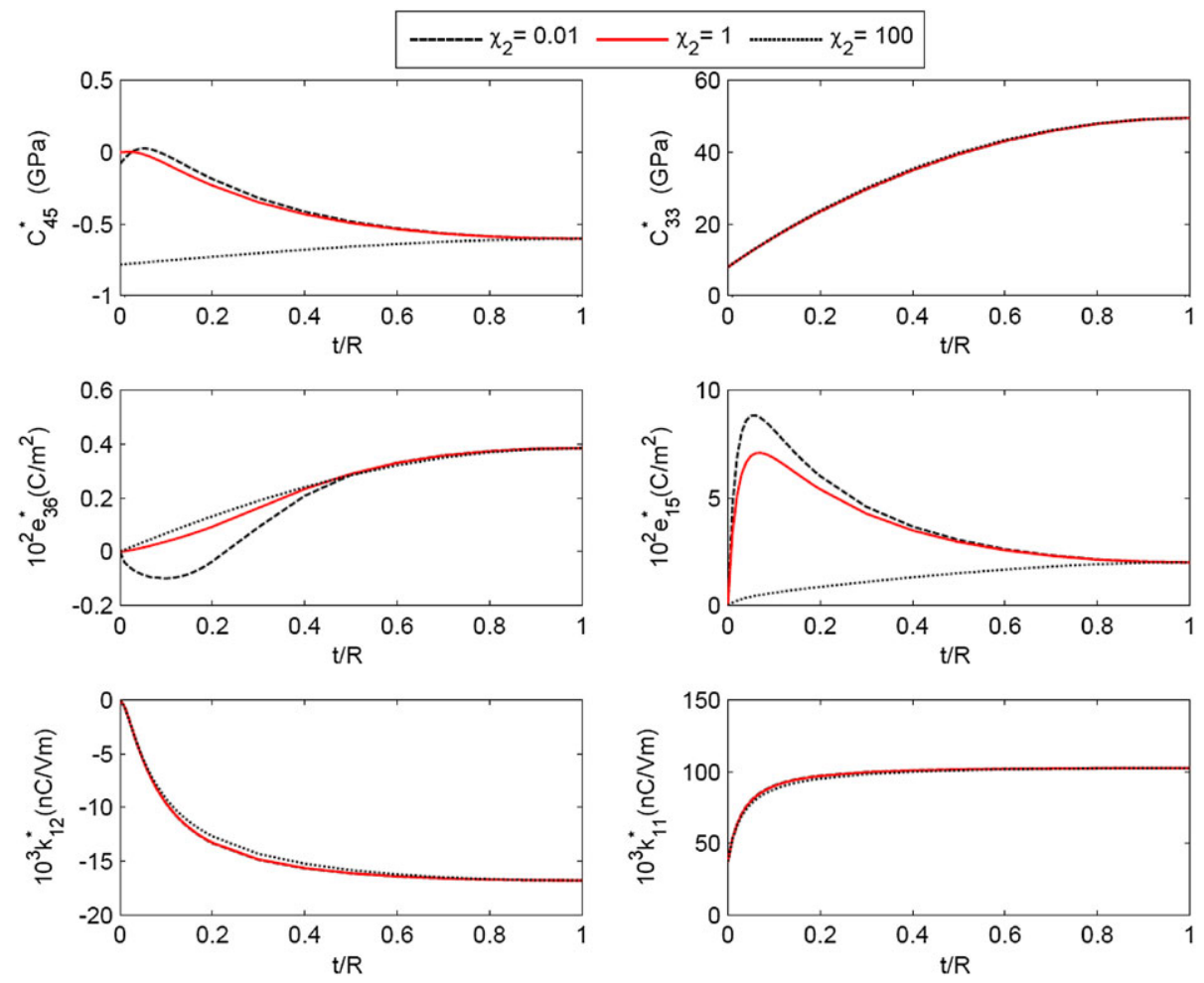

Figure 7. Effective properties for three-phase electro-elastic composites versus the mesophase thickness $t / R$ for different ratios of the shear coefficients.

changing. For instance, the arrays determine the strength or weakness character of the effective properties in the composite. For square cell $\left(\theta=90^{\circ}\right)$, all coefficients in the left sides in Figure 8 are null in contrast with other arrays. In the right sides in Figure 8 , the effective coefficients presented are very similar for the arrays $\theta=90^{\circ}, 75^{\circ}$, but for $\theta=45^{\circ}$ (red line), appears a noticeable difference. Recently, research reports have demonstrated the importance of such composites having parallelepiped arrangements of the fiber distribution in tissues [31] and crystallography [32], and so on. 

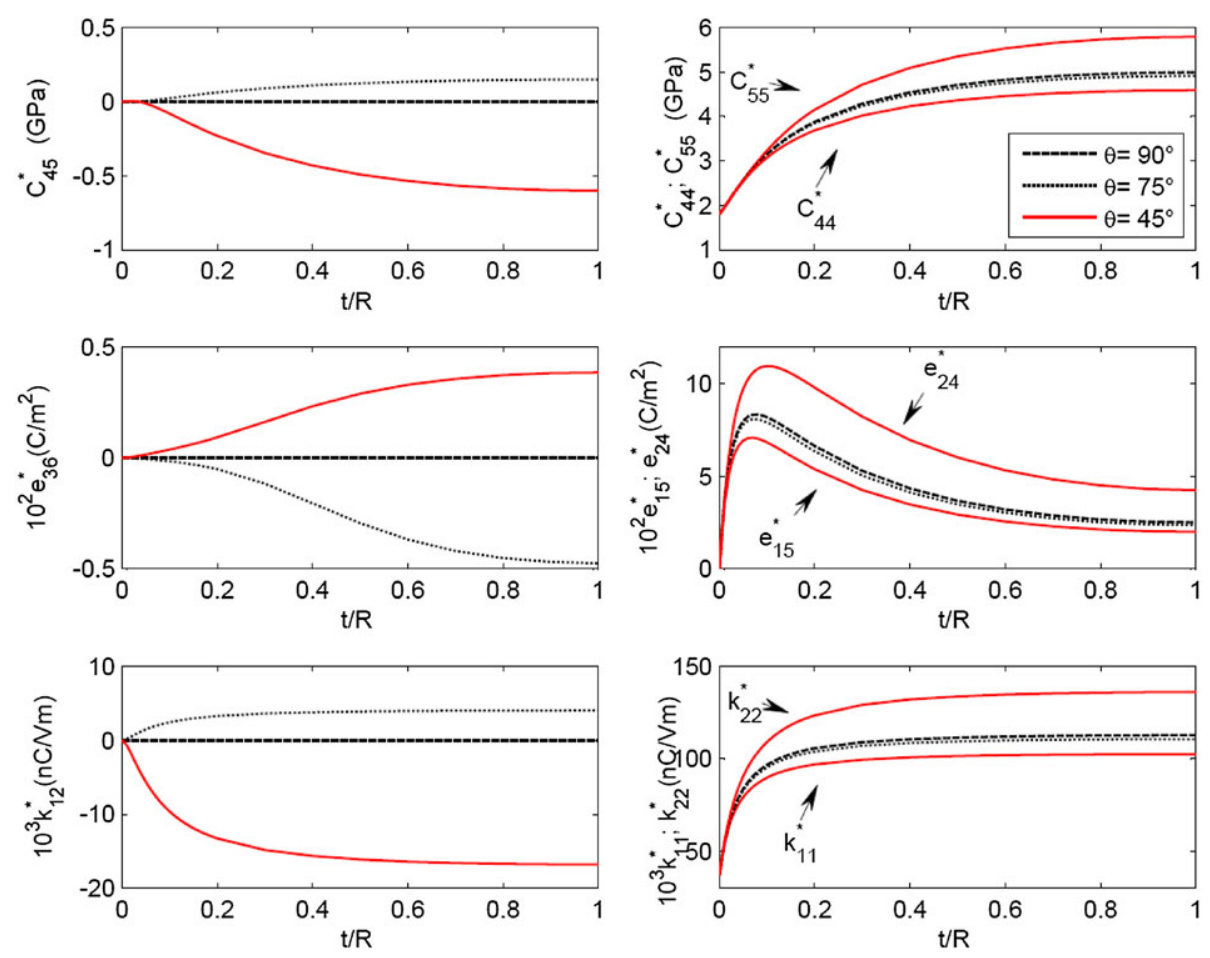

Figure 8. Three different cell arrangements are considered, that is, $\theta=90^{\circ}, 75^{\circ}, 45^{\circ}$ for the study of the influence of parallelepiped arrays on the effective properties behavior.

\section{Conclusions}

In this work, closed analytic expressions for three-phase fibrous periodic composites with parallelepiped cells are derived using two different approaches. The analytical formulae derived for all piezoelectric effective properties have a simple form, and the computational implementation is easy. In comparison with previous publications, a complete characterization of the piezoelectric fiber composite with periodic array is allowable for different situations: (i) considering a thin mesophase, the influence of the perfect and imperfect contact between the matrix and fiber is described; (ii) for the first time, all effective properties for ring-shaped fiber-reinforced piezoelectric composite are calculated and compared with a cylindrical fiber case; (iii) a thick mesophase is studied, and the model illustrated that some piezoelectric properties have an extreme value in comparison with the composite without interphase; and (iv) the numerical proof showed by the applied models (AHM and FEM) are simple and accurate and demonstrated the influence of arrays in the properties of composite. In addition, we can concluded that the influence of the mesophase on the effective moduli can induce a strong anisotropic when the distribution of fibers with different density is taken account and a new coefficient appears and not being when the cell is considered square and hexagonal.

\section{Appendix A}

In order to realize the numerical calculations of the effective coefficient given in (18)-(21), the following linear equations system that characterizes the contact conditions associated with the local problem ${ }_{p 3} L$, should be solved. This system can be rewritten into a new matricial system in terms of $\bar{a}_{p}, a_{p}^{(I)}, \bar{a}_{-p}^{(l)}, \bar{b}_{p}, b_{p}^{(l)}, \bar{b}_{-p}^{(l)}, c_{p}$ and $d_{p}$ which can be solved by Gauss's method.

$$
\begin{gathered}
a_{0} \delta_{1 p}+\sum_{k=1}^{\infty}{ }^{o} a_{k} \eta_{k p}+\bar{a}_{p}-a_{p}^{(l)} \frac{R_{1}^{p}}{R_{2}^{p}}-\bar{a}_{-p}^{(l)}=0 \\
\sqrt{\frac{\tau_{1}}{\lambda_{1}}}\left[b_{0} \delta_{1 p}+\sum_{k=1}^{\infty}{ }^{o} b_{k} \eta_{k p}+\bar{b}_{p}\right]-b_{p}^{(I)} \frac{R_{1}^{p}}{R_{2}^{p}}-\bar{b}_{-p}^{(l)}=0 \\
a_{p}^{(l)}+\bar{a}_{-p}^{(l)} \frac{R_{1}^{p}}{R_{2}^{p}}-c_{p}=0 \\
\sqrt{\lambda_{1} \tau_{1}}\left[b_{p}^{(l)}+\bar{b}_{-p}^{(l)} \frac{R_{1}^{p}}{R_{2}^{p}}\right]-\sqrt{\tau_{1} \lambda_{2}} d_{p}=0
\end{gathered}
$$




$$
\begin{gathered}
a_{0} \delta_{1 p}+\sum_{k=1}^{\infty}{ }^{o} a_{k} \eta_{k p}-\bar{a}_{p}+E_{15}^{(1)}\left[b_{0} \delta_{1 p}+\sum_{k=1}^{\infty}{ }^{o} b_{k} \eta_{k p}-\bar{b}_{p}\right]-\lambda_{1}\left[a_{p}^{(l)}+E_{15}^{(l)} b_{p}^{(l)}\right] \frac{R_{1}^{p}}{R_{2}^{p}}+ \\
+\lambda_{1}\left[\bar{a}_{-p}^{(l)}+E_{15}^{(I)} \bar{b}_{-p}^{(l)}\right]=\left(\lambda_{1}-1\right) R_{1}\left[\delta_{\alpha 1}-i \delta_{\alpha 2}\right] \delta_{1 p} \\
E_{15}^{(1)}\left[a_{0} \delta_{1 p}+\sum_{k=1}^{\infty}{ }^{o} a_{k} \eta_{k p}-\bar{a}_{p}\right]-\left[b_{0} \delta_{1 p}+\sum_{k=1}^{\infty}{ }^{o} b_{k} \eta_{k p}-\bar{b}_{p}\right]-\sqrt{\lambda_{1} \tau_{1}}\left[E_{15}^{(l)} a_{p}^{(l)}-b_{p}^{(l)}\right] \frac{R_{1}^{p}}{R_{2}^{p}}+ \\
+\sqrt{\lambda_{1} \tau_{1}}\left[E_{15}^{(l)} \bar{a}_{-p}^{(l)}-\bar{b}_{-p}^{(l)}\right]=\left(\sqrt{\lambda_{1} \tau_{1}} E_{15}^{(l)}-E_{15}^{(1)}\right) R_{1}\left[\delta_{\alpha 1}-i \delta_{\alpha 2}\right] \delta_{1 p}, \\
\lambda_{1}\left[a_{p}^{(l)}-\bar{a}_{-p}^{(l)} R_{1}^{p}\right]+\lambda_{1}^{p} E_{15}^{(l)}\left[b_{p}^{(l)}-\bar{b}_{-p}^{(l)} \frac{R_{1}^{p}}{R_{2}^{p}}\right]-\lambda_{2}\left[c_{p}+E_{15}^{(2)} d_{p}\right]=\left(\lambda_{2}-\lambda_{1}\right) R_{2}\left[\delta_{\alpha 1}-i \delta_{\alpha 2}\right] \delta_{1 p}, \\
\sqrt{\lambda_{1} \tau_{1}} E_{15}^{(l)}\left[a_{p}^{(l)}-\bar{a}_{-p}^{(l)} \frac{R_{1}^{p}}{R_{2}^{p}}\right]-\sqrt{\lambda_{1} \tau_{1}}\left[b_{p}^{(l)}-\bar{b}_{-p}^{(l)} \frac{R_{1}^{p}}{R_{2}^{p}}\right]-\sqrt{\lambda_{2} \tau_{2}}\left[E_{15}^{(2)} c_{p}-d_{p}\right]= \\
=\left(\sqrt{\lambda_{2} \tau_{2}} E_{15}^{(2)}-\sqrt{\lambda_{1} \tau_{1}} E_{15}^{(l)}\right) R_{2}\left[\delta_{\alpha 1}-i \delta_{\alpha 2}\right] \delta_{1 p} .
\end{gathered}
$$

\section{Appendix B}

The infinite systems in term of the unknown constant $a_{p}$ corresponding to the each local problem ${ }_{q q} L_{12} L$ and ${ }_{3} L$ are the following:

$$
a_{p}+H_{1 p} a_{1}+H_{2 p} \bar{a}_{1}+\sum_{k=1}^{\infty}{ }^{o} W_{k p} a_{k}+\sum_{k=1}^{\infty}{ }^{o} M_{k p} \bar{a}_{k}=T_{\mathscr{F}}
$$

where

$$
\begin{aligned}
H_{1 p} & =B_{p}\left(A_{3} \kappa_{1} \delta_{1 p}-\mathrm{F}_{1}\left(\bar{\eta}_{1 p}-\bar{A}_{1} R_{1}^{2} \delta_{1 p}\right) A_{1}\right) R_{1}^{2}, \\
H_{2 p} & =B_{p}\left(A_{5} \delta_{1 p}-\mathrm{F}_{1}\left(\bar{\eta}_{1 p}-\bar{A}_{1} R_{1}^{2} \delta_{1 p}\right) \bar{A}_{1}\right) R_{1}^{2}, \\
W_{k p} & =B_{p}\left(\mathrm{~F}_{1}\left(\bar{\eta}_{1 p}-\bar{A}_{1} R_{1}^{2} \delta_{1 p}\right) \eta_{k 1}-\mathrm{A}_{2 k} r_{k p}\right), \\
M_{k p} & =B_{p}\left(G_{k p}+\mathrm{F}_{1}\left(\bar{\eta}_{1 p}-\bar{A}_{1} R_{1}^{2} \delta_{1 p}\right) \bar{\eta}_{k 1}\right),
\end{aligned}
$$

and the independent term is

$$
\mathrm{T}_{\mathscr{F}}=\gamma_{1} E_{1} R_{1} \delta_{1 p}-\gamma_{2} B_{p} \mathrm{~F}_{2} R_{1}\left(\bar{\eta}_{1 p}-\bar{A}_{1} R_{1}^{2} \delta_{1 p}\right)-\gamma_{3} B_{p} \mathrm{~F}_{3} R\left(\bar{\eta}_{1 p}-\bar{A}_{1} R_{1}^{2} \delta_{1 p}\right)
$$

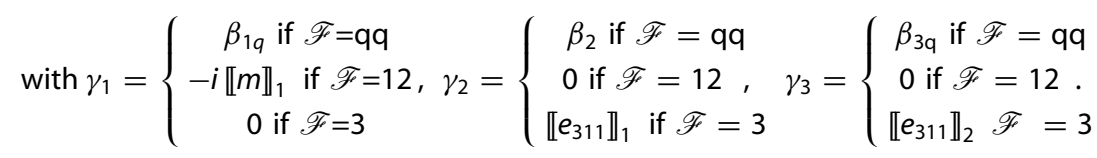

$$
\beta_{1 q}=\frac{C_{22 q q}^{(1)}-C_{22 q q}^{(I)}+C_{11 q q}^{(I)}-C_{11 q q}^{(1)}}{2}, \beta_{2 q}=\frac{C_{11 q q}^{(1)}-C_{11 q q}^{(I)}+C_{22 q q}^{(1)}-C_{22 q q}^{(I)}}{2}, \beta_{3 q}=\frac{C_{11 q q}^{(I)}-C_{11 q q}^{(2)}+C_{22 q q}^{(I)}-C_{22 q q}^{(2)}}{2} .
$$

The magnitudes involved in the system are summarized

$$
\begin{aligned}
g_{k p} & =\left[(p+2)-\left(E_{p}\left(1+\kappa_{l}\right) C_{2 p} / B_{p}\right)\right] \bar{\eta}_{k p+2}+\mathrm{A}_{1 k} k \bar{\eta}_{k+2 p}+k C_{p+k}^{p} R_{1}^{p+k} \bar{T}_{p+k,} \quad C_{k}^{n}=\frac{k !}{n !(k-n) !}, r_{k p} \equiv \sum_{j=1}^{\infty}{ }^{o} \eta_{k j} \bar{\eta}_{j p} \\
T_{k+l} & =\sum_{m, n} \frac{m \bar{w}_{1}+n \bar{w}_{2}}{\left(m w_{1}+n w_{2}\right)^{k+l+1}},(k+l \geq 3,(m, n) \neq(0,0))
\end{aligned}
$$




$$
\begin{aligned}
& \mathrm{A}_{1 p}=1+\Upsilon_{3 p} \chi_{1}\left(1+\kappa_{l}\right)\left(1+\kappa_{1}\right)\left(\chi_{2}-\chi_{1}\right) R_{1}^{2} / p\left(1-\chi_{1}\right) R^{2} \Delta_{p}, \\
& \mathrm{~A}_{2 p}=\left(1+\chi_{1} \kappa_{1}\right) /\left(1-\chi_{1}\right)+\left(\Upsilon_{1 p} \chi_{1}\left(1+\kappa_{l}\right)\left(1+\kappa_{1}\right)\left(\chi_{1} \kappa_{2}+\chi_{2}\right)\left(R_{1} / R\right)^{2 p+4} / \Delta_{p}\left(1-\chi_{1}\right)\right), \\
& B_{p}=\left(1-\chi_{1}\right) /\left(\chi_{1} \kappa_{1}+1-\chi_{1}\left(\kappa_{1}+1\right)\left(\kappa_{l}+1\right)\left(\chi_{2}-\chi_{1}\right)\left(R / R_{1}\right)^{p} \Upsilon_{4 p} / \Delta_{p}\right), \\
& C_{1 p}=\chi_{1}\left(1+\kappa_{1}\right)\left(\chi_{2}-\chi_{1}\right)\left(R / R_{1}\right)^{p} \Upsilon_{4 p} / \Delta_{p}, \\
& C_{2 p}=-\chi_{1}\left(1+\kappa_{1}\right)\left(\chi_{1} \kappa_{2}+\chi_{2}\right)\left(R_{1} / R\right)^{p+2} \Upsilon_{2 p} / \Delta_{p}, \\
& E_{p}=-\left(\chi_{1} \kappa_{1}+1-\chi_{1}\left(\kappa_{1}+1\right)\left(\kappa_{l}+1\right)\left(\chi_{2}-\chi_{1}\right)\left(R / R_{1}\right)^{p} \Upsilon_{4 p} / \Delta_{p}\right)^{-1}, \\
& \Upsilon_{1 p}=\left(\chi_{2}-\chi_{1}\right)\left(\chi_{1}+\kappa_{l}\right)\left(R / R_{1}\right)^{p}+\left(\chi_{1}+\chi_{2} \kappa_{l}\right)\left(\chi_{1}-1\right)\left(R_{1} / R\right)^{p}, \\
& \Upsilon_{2 p}=(p+2)\left(1-\chi_{1}\right)\left(\chi_{2}-\chi_{1}\right)\left[1-\left(R_{1} / R\right)^{2}\right], \\
& \Upsilon_{3 p}=p\left(\chi_{1}-1\right)\left(\chi_{1} \kappa_{2}+\chi_{2}\right)\left(R_{1} / R\right)^{p}\left[1-\left(R_{1} / R\right)^{2}\right], \\
& \Upsilon_{4 p}=\left(\chi_{1} \kappa_{2}-\chi_{2} \kappa_{l}\right)\left(\chi_{1}-1\right)-\left(\chi_{1} \kappa_{2}+\chi_{2}\right)\left(\chi_{1}+\kappa_{l}\right)\left(R_{1} / R\right)^{2 p+4}, \\
& \Delta_{p}=\Upsilon_{1 p} \Upsilon_{4 p}+\Upsilon_{2 p} \Upsilon_{3 p}, \\
& \mathrm{~F}_{0}=\mathrm{F}_{1} /\left(\kappa_{1}-1\right), \quad \mathrm{F}_{1}=\left[\chi_{1}\left(1+\kappa_{1}\right)\left(\kappa_{l}+1\right) \gamma_{0}+2 \Delta_{0}\left(1+\chi_{1} \kappa_{1}-\chi_{1}-\kappa_{l}\right)\right]\left(R_{1} / 4 \alpha_{0} \Delta_{0} R\right), \\
& \mathrm{F}_{2}=\left[\left(1-\kappa_{l}\right) \Delta_{0}+\gamma_{0} \chi_{1}\left(\kappa_{l}+1\right)\left(1-\operatorname{Re}\left(A_{3}\right) R_{1}^{2}\right)\right]\left(R_{1} / 2 \alpha_{0} \Delta_{0} R\right), \mathrm{F}_{3}=\chi_{1}\left(\kappa_{l}+1\right)\left(1-\kappa_{2}\right) R_{1} / 2 R \Delta_{0}, \\
& \Delta_{0}=\gamma_{0} \beta_{0}-\alpha_{0} \delta_{0}, \\
& \gamma_{0}=\chi_{2} \kappa_{l}-\chi_{2}-\chi_{1} \kappa_{2}+\chi_{1}, \quad \delta_{0}=\left(\chi_{1}-\chi_{1} \kappa_{2}-2 \chi_{2}\right)\left(R_{1} / R\right), \\
& \alpha_{0}=\left[\chi_{1}\left(1-\operatorname{Re}\left(A_{3}\right) R_{1}^{2}\right)+\left[1 / 2+\operatorname{Re}\left(A_{3}\right) R_{1}^{2} /\left(\kappa_{1}-1\right)\right]\left(\kappa_{l}-1\right)\right]\left(R_{1} / R\right), \\
& \beta_{0}=\chi_{1}\left(1-\operatorname{Re}\left(A_{3}\right) R_{1}^{2}\right)-1-2 \operatorname{Re}\left(A_{3}\right) R_{1}^{2} /\left(\kappa_{1}-1\right), \\
& \chi_{1}=m_{l} / m_{1} ; \quad \chi_{2}=m_{2} / m_{1}, \\
& \kappa_{s}=3-4 v_{s}^{T}, v_{s}^{T} \text { is the transverse Poisson's ratio ands }=1, l, 2 .
\end{aligned}
$$

\section{Appendix C}

Parameters associated with the analytical expressions of the effective coefficients (22)-(34),

$$
\begin{aligned}
& \left.\left.\left.\mathbf{c}_{1}=\frac{\chi_{1}}{2} \quad P^{-} \quad A_{1} a_{1} R_{1}^{2}-\sum_{k=1}^{\infty}{ }^{o} a_{k} \eta_{k 1}\right)+P^{+} \quad \bar{A}_{1} \bar{a}_{1} R_{1}^{2}-\sum_{k=1}^{\infty}{ }^{o} \bar{a}_{k} \bar{\eta}_{k 1}\right)-P_{1} R_{1} \gamma_{2}+P_{2} R \gamma_{4}\right), \\
& \left.\mathbf{c}_{3}=\frac{1}{\Delta_{1}} \Upsilon_{31} \chi_{1}\left(1+\kappa_{1}\right)\left(\chi_{2}-\chi_{1}\right)\left(R / R_{1}\right) \bar{a}_{1}-\Upsilon_{11} \chi_{1}\left(1+\kappa_{1}\right)\left(\chi_{1} \kappa_{2}+\chi_{2}\right)\left(R_{1} / R\right)^{3} \sum_{k=1}^{\infty}{ }^{o} a_{k} \eta_{k 3}\right), \\
& \left.\mathbf{c}_{-1}=\frac{1}{\Delta_{1}} \Upsilon_{41} \chi_{1}\left(1+\kappa_{1}\right)\left(\chi_{2}-\chi_{1}\right)\left(R / R_{1}\right) a_{1}+\Upsilon_{21} \chi_{1}\left(1+\kappa_{1}\right)\left(\chi_{1} \kappa_{2}+\chi_{2}\right)\left(R_{1} / R\right)^{3} \sum_{k=1}^{\infty}{ }^{o} \bar{a}_{k} \bar{\eta}_{k 3}\right), \\
& \left.\mathbf{d}_{1}=\frac{\chi_{1}\left(1+\kappa_{1}\right)\left(R / R_{1}\right)}{\chi_{1}-1} \quad 1-\frac{\left(\chi_{2}-\chi_{1}\right)\left(3\left(\chi_{1}-1\right) \Upsilon_{31}\left(R_{1} / R\right)^{2}+\left(\chi_{1}+\kappa_{1}\right) \Upsilon_{41}\left(R / R_{1}\right)\right)}{\Delta}\right) \bar{a}_{1}+ \\
& +\frac{\chi_{1}\left(1+\kappa_{1}\right)\left(\chi_{1} \kappa_{2}+\chi_{2}\right)}{\Delta_{1}\left(\chi_{1}-1\right)}\left(R_{1} / R\right)^{3}\left(3\left(\chi_{1}-1\right) \Upsilon_{11}\left(R_{1} / R\right)^{2}-\left(\chi_{1}+\kappa_{l}\right) \Upsilon_{21}\left(R / R_{1}\right)\right) \sum_{k=1}^{\infty}{ }^{o} a_{k} \eta_{k 3}+\frac{R \chi_{1}}{\left(\chi_{1}-1\right)} \boldsymbol{\gamma}_{1}, \\
& \left.\mathbf{d}_{-1}=-\frac{\chi_{1} \gamma_{0}\left(1+\kappa_{1}\right)}{2 \Delta_{0}} \quad A_{1} R_{1}^{2} a_{1}+\bar{A}_{1} R_{1}^{2} \bar{a}_{1}-\sum_{k=1}^{\infty}{ }^{o}\left(a_{k} \eta_{k 1}+\bar{a}_{k} \bar{\eta}_{k 1}\right)\right)+\frac{\gamma_{0} \chi_{1}}{\Delta_{0}}\left(1-\operatorname{Re}\left(A_{3}\right) R_{1}^{2}\right) R_{1} \gamma_{2}-\frac{\alpha_{0} \chi_{1}}{\Delta_{0}}\left(\kappa_{2}-1\right) R \gamma_{3} \\
& \mathbf{P}^{ \pm}=\frac{\delta_{0}\left(1+\kappa_{1}\right)}{2 \Delta_{0}} \pm \frac{\left(\kappa_{1}+1\right)}{\left(\kappa_{l}+1\right)} \frac{R}{R_{1}}+2 i \mathrm{~F}_{1} \frac{\operatorname{Im}\left(A_{3}\right) R R_{1}}{\left(\kappa_{l}+1\right)}, P_{1}=\frac{\delta_{0}\left(1-\operatorname{Re}\left(A_{3}\right) R_{1}^{2}\right)}{\Delta_{0}}+2 i \mathrm{~F}_{2} \frac{\operatorname{Im}\left(A_{3}\right) R R_{1}}{\left(\kappa_{l}+1\right)} \\
& \mathbf{P}_{2}=\frac{\beta_{0}\left(\kappa_{2}-1\right)}{\Delta_{0}}-2 i \mathrm{~F}_{3} \frac{\operatorname{Im}\left(A_{3}\right) R R_{1}}{\left(\kappa_{1}+1\right)}, \\
& \mathbf{c}_{3}=\frac{1}{\Delta_{1}}\left(\Upsilon_{31} \chi_{1}\left(1+\kappa_{1}\right)\left(\chi_{2}-\chi_{1}\right)\left(R / R_{1}\right) \bar{a}_{1}-\Upsilon_{11} \chi_{1}\left(1+\kappa_{1}\right)\left(\chi_{1} \kappa_{2}+\chi_{2}\right)\left(R_{1} / R\right)^{3} \sum_{k=1}^{\infty}{ }^{o} a_{k} \eta_{k 3}\right), \\
& \left.\mathbf{c}_{-1}=\frac{1}{\Delta_{1}} \Upsilon_{41} \chi_{1}\left(1+\kappa_{1}\right)\left(\chi_{2}-\chi_{1}\right)\left(R / R_{1}\right) \mathbf{a}_{1}+\Upsilon_{21} \chi_{1}\left(1+\kappa_{1}\right)\left(\chi_{1} \kappa_{2}+\chi_{2}\right)\left(R_{1} / R\right)^{3} \sum_{k=1}^{\infty}{ }^{o} \bar{a}_{k} \bar{\eta}_{k 3}\right),
\end{aligned}
$$




$$
\begin{aligned}
\mathbf{d}_{1}= & \left.\frac{\chi_{1}\left(1+\kappa_{1}\right)\left(R / R_{1}\right)}{\chi_{1}-1} 1-\frac{\left(\chi_{2}-\chi_{1}\right)\left(3\left(\chi_{1}-1\right) \Upsilon_{31}\left(R_{1} / R\right)^{2}+\left(\chi_{1}+\kappa_{1}\right) \Upsilon_{41}\left(R / R_{1}\right)\right)}{\Delta_{1}}\right) \bar{a}_{1}+ \\
& +\frac{\chi_{1}\left(1+\kappa_{1}\right)\left(\chi_{1} \kappa_{2}+\chi_{2}\right)}{\Delta_{1}\left(\chi_{1}-1\right)}\left(R_{1} / R\right)^{3}\left(3\left(\chi_{1}-1\right) \Upsilon_{11}\left(R_{1} / R\right)^{2}-\left(\chi_{1}+\kappa_{1}\right) \Upsilon_{21}\left(R / R_{1}\right)\right) \sum_{k=1}^{\infty}{ }^{o} a_{k} \eta_{k 3}+\frac{R \chi_{1}}{\left(\chi_{1}-1\right)} \gamma_{1}, \\
\mathbf{d}_{-1}= & \left.-\frac{\chi_{1} \gamma_{0}\left(1+\kappa_{1}\right)}{2 \Delta_{0}} A_{1} R_{1}^{2} a_{1}+\bar{A}_{1} R_{1}^{2} \bar{a}_{1}-\sum_{k=1}^{\infty}{ }^{o}\left(a_{k} \eta_{k 1}+\bar{a}_{k} \bar{\eta}_{k 1}\right)\right)+\frac{\gamma_{0} \chi_{1}}{\Delta_{0}}\left(1-\operatorname{Re}\left(A_{3}\right) R_{1}^{2}\right) R_{1} \gamma_{2}-\frac{\alpha_{0} \chi_{1}}{\Delta_{0}}\left(\kappa_{2}-1\right) R \gamma_{3} .
\end{aligned}
$$

\section{Acknowledgement}

The funding of CONACYT project number 129658 and Coordinación de la Investigación Cientifica (COIC-STIA-306-15 (7178-7179)) UNAM is gratefully acknowledged. Y. Espinosa-Almeyda would like to thank CONACYT for a scholarship funding. The authors gratefully acknowledge the project SHICHAN, supported by FSP (Cooperation Scientifique Franco-Cubaine) Cuba 2011-26, PROJET N 29935XH and to the project Composite Materials from University of Havana. Author AC acknowledges the financial support received through CNPQ (PQ - 392298/2012-6). The author RRR wants to thank the PROPPI and Department of International Relations - DRI - Universidade Federal Fluminense - UFF, for supporting the development of this work during his stay in the Computer Institute at the Universidade Federal Fluminese (Project Edital PI-UFF 2014). Thanks to Departamento de Matemáticas y Mecánica, IIMAS-UNAM, for its support and Ramiro Chávez Tovar and Ana Pérez Arteaga for computational assistance.

\section{References}

1. Benveniste Y. Exact results in the micromechanics of fibrous piezoelectric composites exhibiting pyroelectricity. Proceedings of The Royal Society of London 1993; A441:59-81.

2. Zhang TY, Qian CF, Tong P. Linear electroelastic analysis of a cavity or a crack in a piezoelectric material. International Journal of Solids and Structures 1998; 35:2121-2149.

3. Jiang $\mathrm{CP}$, Tong $\mathrm{ZH}$, Cheung YK. A generalized self-consistent method for piezoelectric fiber reinforced composites under antiplane shear. Mechanics of Materials 2001a; 33:295-308.

4. Dinzart F, Sabar H. Electroelastic behavior of piezoelectric composites with coated reinforcements: micromechanical approach and applications. International Journal of Solids and Structures 2009; 46:3556-3564.

5. Rodríguez-Ramos R, Guinovart-Díaz R, López-Realpozo JC, Bravo-Castillero J, Otero JA, Sabina FJ, Lebon F. Effective properties of periodic fibrous electro-elastic composites with mechanic imperfect contact condition. International Journal of Mechanical Sciences 2013; 73:1-13.

6. Xu Y, Xiao J, Jia J, Qiu P, Zhao Q. An analytical method for piezoelectric composites containing doubly periodic piezoelectric fibers with ring-shaped cross-section under antiplane shear and its application. Computation Materials Science 2014; 88:7-13.

7. Eynbeygi M, Aghdam MM. A micromechanical study on the electro-elastic behaviour of piezoelectric fiber-reinforced composites using the elementfree Galerkin method. Acta Mechanica 2015; 226(9):3177-3194.

8. Wang X, Pan E. Two-dimensional Eshelby's problem for two imperfectly bonded piezoelectric half-planes. International Journal of Solids and Structures 2010; 47:148-160.

9. Gu ST, He QC, Pensée V. Homogenization of fibrous piezoelectric composites with general imperfect interfaces under anti-plane mechanical and in-plane electrical loadings. Mechanics of Materials 2015; 88:12-29.

10. Shen MH, Chen SN, Chen FM. Piezoelectric study on confocally multicoated elliptical inclusion. International Journal of Engineering Science 2005; 43:1299-1312.

11. Shiah YC, Huang $\mathrm{CH}$, Huang JH. Electromechanical responses of optical fibers with piezoelectric coatings. Journal of the Chinese Institute of Engineers 2006; 29(5):893-902.

12. Xiao JH, Xu YL, Zhang FC. Evaluation of effective electroelastic properties of piezoelectric coated nano-inclusion composites with interface effect under antiplane shear. International Journal of Engineering Science 2013; 69:61-68.

13. Jiang CP, Cheung YK. An exact solution for the three-phase piezoelectric cylinder model under antiplane shear and its applications to piezoelectric composites. International Journal of Solids and Structures 2001; 38:4777-4796.

14. Guinovart-Díaz R, Rodríguez-Ramos R, Bravo-Castillero J, Sabina FJ, Maugin GA. Closed-form thermoelastic moduli of a periodic three-phase fiberreinforced composite. Journal of Thermal Stresses 2005; 28:1067-1093.

15. Guinovart-Díaz R, Rodríguez-Ramos R, Bravo-Castillero J, Sabina FJ, Camacho-Montes H. Electro-mechanical moduli of three-phase fiber composites. Materials Letters 2008; 62:2385-2387.

16. Yan $\mathrm{P}$, Jiang CP, Song F. An eigenfunction expansion-variational method for the anti-plane electroelastic behavior of three-phase fiber composites. Mechanics of Materials 2011; 43:586-597.

17. Sudak LJ. Effect of an interphase layer on the electroelastic stresses within a three-phase elliptic inclusion. International Journal of Engineering Science 2003; 41:1019-1039.

18. Yang BH, Gao CF. Anti-plane electro-elastic fields in an infinite matrix with $\mathrm{N}$ coated-piezoelectric inclusions. Composites Science and Technology 2009; 69:2668-2674.

19. Rodríguez-Ramos R, Guinovart-Díaz R, López-Realpozo JC, Bravo-Castillero J, Sabina FJ, Lebon F, Dumont S, Würkner M, Berger H, Gabbert U. Characterization of piezoelectric composites with mechanical and electrical imperfect contacts. Journal of Composite Materials 2016; 50(12): 1603-1625.

20. Nitsche JA. Convergence of nonconforming methods (finite element solution for Dirichlet problem). In Mathematical Aspects of Finite Elements in Partial Differential Equations, De Boor C (ed). Academic Press: New York, 1974; 15-53.

21. Becker R, Hansbo P, Stenberg R. A finite element method for domain decomposition with non-matching grids. ESAIM: M2AN 2010; 37(1):209-225. 
22. Dumont S, Lebon F, Rizzoni R. An asymptotic approach to the adhesion of thin stiff films. Mechanics Research Communications 2014; 58:24-35.

23. Guinovart-Díaz R, Rodríguez-Ramos R, Bravo-Castillero J, Sabina FJ. Modeling of three-phase fibrous composite using the asymptotic homogenization method. Mechanics of Advanced Materials and Structures 2003; 10(4):319-333.

24. Kari S, Berger H, Gabbert U, Guinovart-Díaz R, Bravo-Castillero J, Rodríguez-Ramos R. Composites Science and Technology 2008; 68:684-691.

25. Sevostianov I, Rodríguez-Ramos R, Guinovart-Díaz R, Bravo-Castillero J, Sabina FJ. Connections between different models describing imperfect interfaces in periodic fiber-reinforced composites. International Journal of Solids and Structures 2012; 49:1518-1525.

26. Rodríguez-Ramos R, Sabina FJ, Guinovart-Díaz R, Bravo-Castillero J. Closed-form expressions for the effective coefficients of a fiber-reinforced composite with transversely isotropic constituents - I. Elastic and square symmetry. Mechanics of Materials 2001; 33:223-235.

27. Bakhvalov NS, Panasenko GP. Homogenization Averaging Processes in Periodic Media. Kluwer Academic Publishers: Kluwer, 1989.

28. Bravo-Castillero J, Guinovart-Díaz R, Sabina FJ, Rodríguez-Ramos R. Closed form expressions for the effective coefficients of a fiber-reinforced composite with transversely isotropic constituents-II. Piezoelectric and square symmetry. Mechanics of Materials 2001; 33(3):237-48.

29. Sabina FJ, Rodríguez-Ramos R, Bravo-Castillero J, Guinovart-Díaz R. Closed-form expressions for the effective coefficients of fibre-reinforced composite with transversely isotropic constituents-II: piezoelectric and hexagonal symmetry. Journal of the Mechanics and Physics of Solids 2001; 49: 1463-1479.

30. Pastor J. Homogenization of linear piezoelectric media. Mechanics Research Communications 1997; 24(1):145-150.

31. Karamuk E, Mayer J, Raeber G. Tissue engineered composite of a woven fabric scaffold with tendon cells, response on mechanical simulation in vitro. Composites Science and Technology 2004; 64:885-891.

32. Olsson PO, Kihlborg L. Three-dimensional effects in multislice image calculations for oblique cells. Ultramicroscopy 1989; 27:387-400. 